\title{
Molecular gas during the post-starburst phase: low gas fractions in green-valley Seyfert post-starburst galaxies
}

\author{
Hassen M. Yesuf, ${ }^{1 \star}$ K. Decker French, ${ }^{2}$ S. M. Faber ${ }^{1}$ and David C. Koo ${ }^{1}$ \\ ${ }^{1}$ University of California Observatories and the Department of Astronomy \& Astrophysics, University of California, Santa Cruz, CA 95064, USA \\ ${ }^{2}$ Steward Observatory, University of Arizona, 933 North Cherry Avenue, Tucson, AZ 85721, USA
}

Accepted 2017 April 26. Received 2017 April 26; in original form 2017 February 8

\begin{abstract}
Post-starbursts (PSBs) are candidate for rapidly transitioning from starbursting to quiescent galaxies. We study the molecular gas evolution of PSBs at $z \sim 0.03-0.2$. We undertook new CO (2-1) observations of 22 Seyfert PSB candidates using the Arizona Radio Observatory Submillimeter Telescope. This sample complements previous samples of PSBs by including green-valley PSBs with Seyfert-like emission, allowing us to analyse for the first time the molecular gas properties of 116 PSBs with a variety of AGN properties. The distribution of molecular gas to stellar mass fractions in PSBs is significantly different from normal starforming galaxies in the CO Legacy Database (COLD) GASS survey. The combined samples of PSBs with Seyfert-like emission line ratios have a gas fraction distribution that is even more significantly different and is broader $(\sim 0.03-0.3)$. Most of them have lower gas fractions than normal star-forming galaxies. We find a highly significant correlation between the WISE 12 and $4.6 \mu \mathrm{m}$ flux ratios and molecular gas fractions in both PSBs and normal galaxies. We detect molecular gas in 27 per cent of our Seyfert PSBs. Taking into account the upper limits, the mean and the dispersion of the distribution of the gas fraction in our Seyfert PSB sample are much smaller $(\mu=0.025, \sigma=0.018)$ than previous samples of Seyfert PSBs or PSBs in general $(\mu \sim 0.1-0.2, \sigma \sim 0.1-0.2)$.
\end{abstract}

Key words: ISM: molecules-galaxies: active-galaxies: evolution-galaxies: nucleigalaxies: Seyfert-galaxies: starburst.

\section{INTRODUCTION}

Despite extensive observational and theoretical studies, the physical mechanisms that regulate the star formation rates (SFRs) of galaxies are still poorly understood. Star formation quenching, by yet unknown mechanisms, causes star-forming galaxies to migrate to the 'red sequence' (Gladders et al. 1998; Faber et al. 2007). One likely such formation of mechanism of the red sequence is the transformation of star-forming, disc-dominated, gas-rich galaxies into early types via mergers and their associated feedback (Toomre \& Toomre 1972; Hopkins et al. 2006).

Post-starburst (PSB) galaxies are candidate post-merger objects, rapidly transitioning from the blue cloud to the red sequence (e.g. Dressler \& Gunn 1983; Couch \& Sharples 1987; Zabludoff et al. 1996; Wild et al. 2009; Snyder et al. 2011; Yesuf et al. 2014; Pawlik et al. 2016). In their quiescent phase, their spectra reveal little-to-no current star formation, but a substantial burst of star formation before an abrupt cessation $\sim 1 \mathrm{Gyr}$ ago, long enough for the ionizing O\&B stars to evolve away, but recent enough for A stars to domi- nate the stellar light. Recent observational efforts have enlarged the traditional definition of PSB to include a more complete and less biased sample of galaxies with ongoing star formation or/and AGN activity (Wild, Heckman \& Charlot 2010; Alatalo et al. 2014; Yesuf et al. 2014). With the identification of this sample, which spans the entire starburst-to-quenched PSB evolutionary sequence, better constraints on theoretical models of galaxy evolution have started to emerge. One of the firm constraints is the observed time delay between the starburst phase and the AGN activity by about $200 \mathrm{Myr}$ (Wild et al. 2010; Yesuf et al. 2014). This time delay suggests that AGNs are not primarily responsible for the original quenching of starbursts but may be responsible for keeping star formation at a low level by removing gas and dust during the PSB phase. The other emerging but contested phenomenon is molecular gas evolution after the starburst has ended (Schawinski et al. 2009; Rowlands et al. 2015). This work aims to further examine the molecular gas evolution along the starburst sequence using both new and existing data, by adding molecular gas observations from green-valley Seyfert PSBs, which were excluded from other PSB samples.

In simulations of gas-rich mergers, gas is funnelled to galaxy centres, powering intense nuclear starbursts and obscured nuclear AGN activity. At the end of the starburst, the leftover gas and dust are 
cleared out due to feedback from the AGN (e.g. Sanders et al. 1988; Barnes \& Hernquist 1991; Silk \& Rees 1998; Di Matteo, Springel \& Hernquist 2005; Springel, Di Matteo \& Hernquist 2005; Hopkins et al. 2006, 2008; Kaviraj et al. 2007; Wild et al. 2009; Snyder et al. 2011; Cen 2012). For instance, Narayanan et al. (2008) found that galactic winds are a natural consequence of merger-induced star formation and black hole growth. In their simulated galaxies, the galactic winds can entrain molecular gas of $\sim 10^{8}-10^{9} \mathrm{M}_{\odot}$, which, the authors showed, should be observable in $\mathrm{CO}$ emission. The molecular gas entrained in the winds driven by AGN is predicted to be longer lived than the gas entrained solely in starburst-driven winds. The wind velocities in the simulated galaxies with AGN feedback can reach close to 2.5 times the circular velocity. Thus, making AGN feedback a viable mechanism to get rid of a residual gas and dust at the end of a starburst.

Despite its theoretical appeal, the evidence that connects AGN activity with the end of star formation in galaxies has been elusive, with evidence both for (Schawinski et al. 2009; Alatalo et al. 2011; Cicone et al. 2014; García-Burillo et al. 2014) and against (Fabello et al. 2011; French et al. 2015; Geréb et al. 2015; Rowlands et al. 2015; Alatalo et al. 2016).

Now we review previous works on molecular gas contents of PSBs and AGN. Using Institut de Radioastronomie Millimétrique (IRAM) CO observations, Rowlands et al. (2015) investigated the evolution of molecular gas and dust properties in 11 PSBs on the starburst-to-quenched PSB sequence at $z \sim 0.03$. Two of these PSBs are Seyfert galaxies while the rest are either star-forming or composite galaxies of star formation and AGN activity. 10/11 of the PSBs were detected in the $\mathrm{CO}(1-0)$ transition and $9 / 11$ of the PSBs were detected in $\mathrm{CO}(2-1)$ transition. The gas and dust contents, the star formation efficiency and the gas depletion time of majority of these PSBs are similar to those of local star-forming spiral galaxies (Saintonge et al. 2011; Boselli et al. 2014) and gas-rich elliptical galaxies (Young et al. 2011; Davis et al. 2014). In addition, the authors found a decrease in dust temperature with the starburst age but they did not find evidence for dust heating by AGN at late times.

Similarly, Alatalo et al. (2016) studied 52 PSBs with shock signatures at $z=0.02-0.2$ using IRAM and Combined Array for Research in Millimeter-Wave Astronomy (CARMA). About half of these PSBs are at $z>0.1,14 / 52$ are Seyferts and 90 per cent have CO (1-0) detections. The molecular gas properties of these PSBs are also similar to those of normal star-forming galaxies. More than 80 per cent of the PSBs in Alatalo et al. (2016) and Rowlands et al. (2015) samples are located in the blue cloud.

French et al. (2015) studied 32 PSBs in the green valley at $z=0.01-0.12$ using the IRAM $30 \mathrm{~m}$ and the Submillimeter Telescope (SMT). Almost all of these PSBs have signatures mimicking low-ionization nuclear emission line regions (LINERs), and 53 per cent of them have $\mathrm{CO}$ detections. Those detected in $\mathrm{CO}$ have gas masses and gas-to-stellar mass fractions comparable to those of star-forming galaxies while the non-detected PSBs have gas fractions more consistent with those observed in quiescent galaxies.

The three aforementioned studies on the molecular gas contents of PSBs suggested that the end of starburst in these galaxies cannot be ascribed to a complete exhaustion or removal or destruction of molecular gas. The studies also suggested that multiple episodes of starburst or/and AGN activities may be needed for the eventual migration of these galaxies to the red sequence and that a transition time longer than 1 Gyr may be needed for this migration to take place.

Saintonge et al. (2012) found that, among the gas-rich, discdominated galaxy population, those that are ongoing mergers or are morphologically disturbed have the shortest molecular gas deple- tion times. They found no link between the presence of AGN and the long depletion times observed in bulge-dominated galaxies. Even though their AGN sample has lower molecular gas fractions than the control sample matched in NUV $-r$ colour and stellar mass surface density, the depletion times of the two populations are similar. More than 90 per cent of the AGN studied by Saintonge et al. (2012) are not Seyferts, and instead are LINERs.

In contrast, Schawinski et al. (2009) presented evidence that AGN are responsible for the destruction of molecular gas in morphologically early-type galaxies at $z \sim 0.05$. Their sample included 10 star-forming galaxies, 10 star formation and AGN composite galaxies and 4 Seyfert galaxies. The galaxies studied by Schawinski et al. (2009) are not PSBs but might have experienced mild recent star formation (see Schawinski et al. 2007). The authors found that the molecular gas mass drops significantly $200 \mathrm{Myr}$ after a recent star formation in the composite galaxies, and none of their Seyferts have $\mathrm{CO}$ detections. The authors interpreted their observations as evidence for a destruction of molecular gas and for a suppression of residual star formation by low-luminosity AGN. Likewise, many studies have reported molecular outflows with high mass-outflow rates as evidence for AGN feedback in non-PSB AGN host galaxies (e.g. Feruglio et al. 2010; Fischer et al. 2010; Sturm et al. 2011; Spoon et al. 2013; Veilleux et al. 2013; Cicone et al. 2014; GarcíaBurillo et al. 2014; Sun et al. 2014).

In this work, we study molecular gas in 22 green-valley, Seyfert PSBs candidates using SMT CO (2-1) observations, in combination with an existing sample of 94 PSBs from the literature. Our observations were motivated by French et al. (2015) and Rowlands et al. (2015) and were designed to be complementary to the samples in these two works. Our sample is also complementary to the recently published sample of shocked PSB galaxies (Alatalo et al. 2016), which contains 14 galaxies with Seyfert-like emission line ratios, which are mainly located in the blue cloud. Our sample is comparable in number to the existing sample of Seyfert PSBs but represents those in the UV-optical green valley. When combined with other samples, our sample is indispensable in sampling PSBs with a variety of AGN properties.

\subsection{Simple energetic argument for no feedback or delayed AGN feedback after the molecular gas fraction is $\lesssim 4$ per cent}

In this section, we present the condition required for momentumdriven AGN wind to clear gas in a galactic disc or very close to the galaxy, following Silk \& Nusser (2010). These authors argued, from the condition they derived, that AGN cannot supply enough momentum in radiation to unbind gas out of haloes of galaxies. They theoretically estimated the total gas mass within a dark matter halo assuming a gas-to-total mass fraction of 10 per cent. In this work, we measure the molecular gas mass-to-the stellar mass fraction, $f_{\mathrm{H}_{2}}=\frac{M_{\mathrm{H}_{2}}}{M_{\star}}$, in the discs of our galaxies (or very close to the vicinities of the galaxies). Our estimated condition is local, while Silk \& Nusser (2010) were interested if the AGN-driven winds eventually escape from the haloes of galaxies. We recast their derivation in terms of molecular gas fraction to show that AGN may only impart sufficient momentum to clear galactic discs once the molecular gas fraction is below about $\lesssim 4$ per cent, perhaps explaining the delayed AGN molecular gas destruction hinted in this work, and the minor role of AGN in quenching starbursts (e.g. Wild et al. 2010; Yesuf et al. 2014). Note that starbursts have molecular gas fractions of 20 30 per cent while normal star-forming galaxies have gas fractions of about 10 per cent. 
Let us assume an isothermal sphere galaxy. Within a halo radius $r$, its total mass is $M_{\mathrm{gal}}=2 \sigma^{2} r / G$, where $G$ is Newton's gravitational constant and $\sigma$ is the velocity dispersion.

The total gas fraction, $f_{\text {gas }}$, in terms of the molecular hydrogen gas fraction is

$f_{\text {gas }}=M_{\text {gas }} / M_{\star}=\left(M_{\mathrm{HI}}+M_{\mathrm{H}_{2}}\right) / M_{\star}=\left(1+M_{\mathrm{HI}} / M_{\mathrm{H}_{2}}\right) f_{\mathrm{H}_{2}}$.

If $f_{\star}$ is the ratio between stellar mass and the total (halo) mass of the galaxy, $f_{\star}=\frac{M_{\star}}{M_{\text {gal }}}$, then

$M_{\mathrm{gas}}=\left(1+M_{\mathrm{HI}} / M_{\mathrm{H}_{2}}\right) f_{\mathrm{H}_{2}} f_{\star} M_{\mathrm{gal}}$.

Assuming that the AGN luminosity, $L$, is a fraction of the Eddington luminosity, $L=\lambda_{\mathrm{Edd}} L_{\mathrm{Edd}}$, where $\lambda_{\mathrm{Edd}}$ is the Eddington ratio and the Eddington luminosity is $L_{\mathrm{Edd}}=4 \pi G M_{\mathrm{BH}} m_{\mathrm{p}} c / \sigma_{\mathrm{T}}$. If the radiation pressure balances gravitational force (Murray, Quataert \& Thompson 2005),

$\lambda_{\mathrm{Edd}} L_{\mathrm{Edd}} / c=G M_{\mathrm{gal}} M_{\mathrm{gas}} / r^{2}$

$\Rightarrow M_{\mathrm{BH}}=\left(1+M_{\mathrm{HI}} / M_{\mathrm{H}_{2}}\right) f_{\mathrm{H}_{2}} \frac{f_{\star}}{\lambda_{\mathrm{Edd}}} \frac{\sigma_{\mathrm{T}}}{m_{p}} \frac{\sigma^{4}}{\pi G^{2}}$

which has similar scaling as the observed $M_{\mathrm{BH}}-\sigma$ relation.

The total energy radiated by the black hole, $E_{\text {out }}$, while driving the gas at radius $R$ near the vicinity of the host galaxy by the radiation pressure is $E_{\text {out }}=L \int_{R_{0}}^{R} 1 / v(r) \mathrm{d} r>L \cdot\left(R-R_{0}\right) / v_{\mathrm{e}}(R)$, where $v_{\mathrm{e}}(R)$ is the escape speed at the radius $R$. For an isothermal sphere truncated at $R_{\max }, v_{\mathrm{e}}(R)=2 \sigma \sqrt{1+\ln R_{\max } / R}$. The work done by radiation pressure in moving the gas from $R_{0}$ to $R$ must be greater than the kinetic energy required for the gas to the escape, $L \cdot\left(R-R_{0}\right) / c>1 / 2 M_{\mathrm{gas}} v_{\mathrm{e}}^{2}(R)$. Thus,

$E_{\text {out }}>c / 2 M_{\text {gas }} v_{\mathrm{e}}(R)>M_{\mathrm{gas}} c \sigma \sqrt{1+\ln R_{\max } / R}$

$E_{\text {out }}>\left(1+M_{\mathrm{HI}} / M_{\mathrm{H}_{2}}\right) f_{\mathrm{H}_{2}} M_{\star} c \sigma \sqrt{1+\ln R_{\max } / R}$.

The accretion energy of the black hole must also satisfy

$\eta M_{\mathrm{BH}} c^{2}>\left(1+M_{\mathrm{HI}} / M_{\mathrm{H}_{2}}\right) f_{\mathrm{H}_{2}} M_{\star} c \sigma \sqrt{1+\ln R_{\max } / R}$.

Dividing both sides by $M_{\star}$ leads to the condition

$f_{\mathrm{H}_{2}}<\frac{\eta}{\left(1+M_{\mathrm{HI}} / M_{\mathrm{H}_{2}}\right)} \frac{M_{\mathrm{BH}}}{M_{\text {bulge }}} \frac{M_{\text {bulge }}}{M_{\star}} \frac{c}{\sigma \sqrt{1+\ln R_{\max } / R}}$.

Using the observed ratio $M_{\mathrm{BH}} / M_{\text {bulge }} \sim 3 \times 10^{-3}$ (e.g. Kormendy 2013), $\eta=0.1, M_{\text {bulge }} / M_{\star}=0.5, \sigma=150 \mathrm{~km} \mathrm{~s}^{-1}$, $\left(1+M_{\mathrm{HI}} / M_{\mathrm{H}_{2}}\right) \sim 4$ (Saintonge et al. 2011) and $R_{\max } / R=10$ gives

$$
\begin{aligned}
f_{\mathrm{H}_{2}} \lesssim & 0.04\left(\frac{M_{\text {bulge }}}{M_{\star}} / 0.5\right)\left(\frac{150 \mathrm{~km} \mathrm{~s}^{-1}}{\sigma}\right)\left(\frac{4}{1+M_{\mathrm{HI}} / M_{\mathrm{H}_{2}}}\right) \\
& \times\left(\frac{1.82}{\sqrt{1+\ln R_{\max } / R}}\right) .
\end{aligned}
$$

The above assumed values are reasonable for PSB galaxies. Similar assumption of $R_{\max } / R=10$ is also made by Rupke, Veilleux $\&$ Sanders (2002) in studying galactic winds in local ultraluminous infrared galaxies. The gas measurements are made within $R \sim 10$ $20 \mathrm{kpc}$ of our galaxies while their dark matter haloes may extend to few $100 \mathrm{kpc} . R_{\max } / R$ may range between 10 and 100 . Adopting $R_{\max } / R=100$ instead will only change our $f_{\mathrm{H}_{2}}$ limit by a factor of 1.3. Only few per cent of the AGN luminosity is expected to couple to the gas. Therefore, the above lower limit is very liberal estimate since it assumes 100 per cent feedback efficiency. 5 per cent feedback efficiency is often used in AGN feedback models (e.g. Scannapieco \& Oh 2004; Di Matteo et al. 2005; Zubovas \& King 2012). Therefore, our estimate implies that that AGN feedback may only be effective in late-stage PSBs after the molecular gas fraction is below $\lesssim 4$ per cent.

The rest of the paper is organized as follows: Section 2 presents the sample selection. Section 3 presents overview of statistical methods used in the paper. Section 4 presents the main results. Section 5 discusses our sample in comparison with other samples of PSBs. Section 6 summarizes the main findings of this work. Appendix A provides ancillary information on how our sample relates to existing samples of PSBs with molecular gas measurements. We assume $\left(\Omega_{\mathrm{m}}, \Omega_{\Lambda}, h\right)=(0.3,0.7,0.7)$ cosmology.

\section{SAMPLE SELECTION AND OBSERVATIONS}

\subsection{Sample selection}

Using the cross-matched catalogue of Sloan Digital Sky Survey (SDSS), GALEX and WISE (Martin et al. 2005; Wright et al. 2010; Aihara et al. 2011; Yesuf et al. 2014), we select a sample of 22 transition PSB Seyfert galaxy candidates based on the evolutionary path that starbursts and PSBs follow in the dust-corrected NUV $-g$ colour, $\mathrm{H} \delta$ absorption equivalent width and the $4000 \AA$ break (Yesuf et al. 2014), and based on the BPT line ratio AGN diagnostic (Baldwin, Phillips \& Terlevich 1981; Kewley et al. 2001; Kauffmann et al. 2003), as shown in Fig. $1 . D_{n}(4000)$ probes the average temperature of the stars responsible for the continuum emission and is a good indicator of the mean stellar age (Bruzual 1983; Balogh et al. 1999). The combination NUV $-g$ colour with $\mathrm{H} \delta$ and $D_{n}(4000)$ is useful in identifying late-stage PSB galaxies, which are outliers at intermediate age from loci of galaxies with regular (continuous) star formation histories. As discussed in Yesuf et al. (2014), we acquired the measurements for physical parameters such as stellar masses and spectral indices from the publicly available catalogues on SDSS website ${ }^{1}$ (Aihara et al. 2011). Our sample is restricted to galaxies with redshift, $z<0.06$, stellar mass, $M>10^{10} \mathrm{M}_{\odot}$, $(\mathrm{NUV}-g)_{\mathrm{dc}}>2.2$ and $D_{n}(4000)_{\mathrm{dc}}<1.55$ and $\mathrm{H} \delta>1 \AA$. The subscript 'dc' denotes dust correction. We use the $\mathrm{H} \alpha / \mathrm{H} \beta$ flux ratio with two-component dust attenuation model of Charlot \& Fall (2000) to correct for dust attenuation of the nebular emission lines, and the empirical relationship between the emission line and continuum optical depths found in Wild et al. (2011) to correct the continuum fluxes (for more details, see Yesuf et al. 2014). Two candidate PSB galaxies with $\mathrm{CO}$ observations are removed from the main analyses because they do not satisfy the above cuts. TPSB12 is removed because it has $\mathrm{H} \delta<1 \AA$ while TPSB13 is removed because it has (NUV $-g)_{\mathrm{dc}}<2$ and it is also an ongoing merger. Including these two PSBs instead does not change the main conclusions. Furthermore, note that in Fig. 1 we only show galaxies with NUV detections (Yesuf et al. 2014) in previous samples of PSBs (French et al. 2015; Rowlands et al. 2015; Alatalo et al. 2016). In later analyses, this restriction is not required.

The redshift cut, $z<0.06$, was imposed due to the sensitivity of the SMT to achieve the desired signal-to-noise ratio in 6-8 h. This severely limited the number of Seyferts available for the observation. In addition, in the second year of the observation, the sample was restricted to be above a declination of $35^{\circ}$ (away from the Sun avoidance zone for the SMT and the bulge of the Galaxy), further limiting the observable sample. Therefore, we did not impose the $\mathrm{H} \delta>4 \AA$ absorption cut that was done in the original

\footnotetext{
${ }^{1}$ https://www.sdss3.org/dr8/spectro/galspec.php
} 

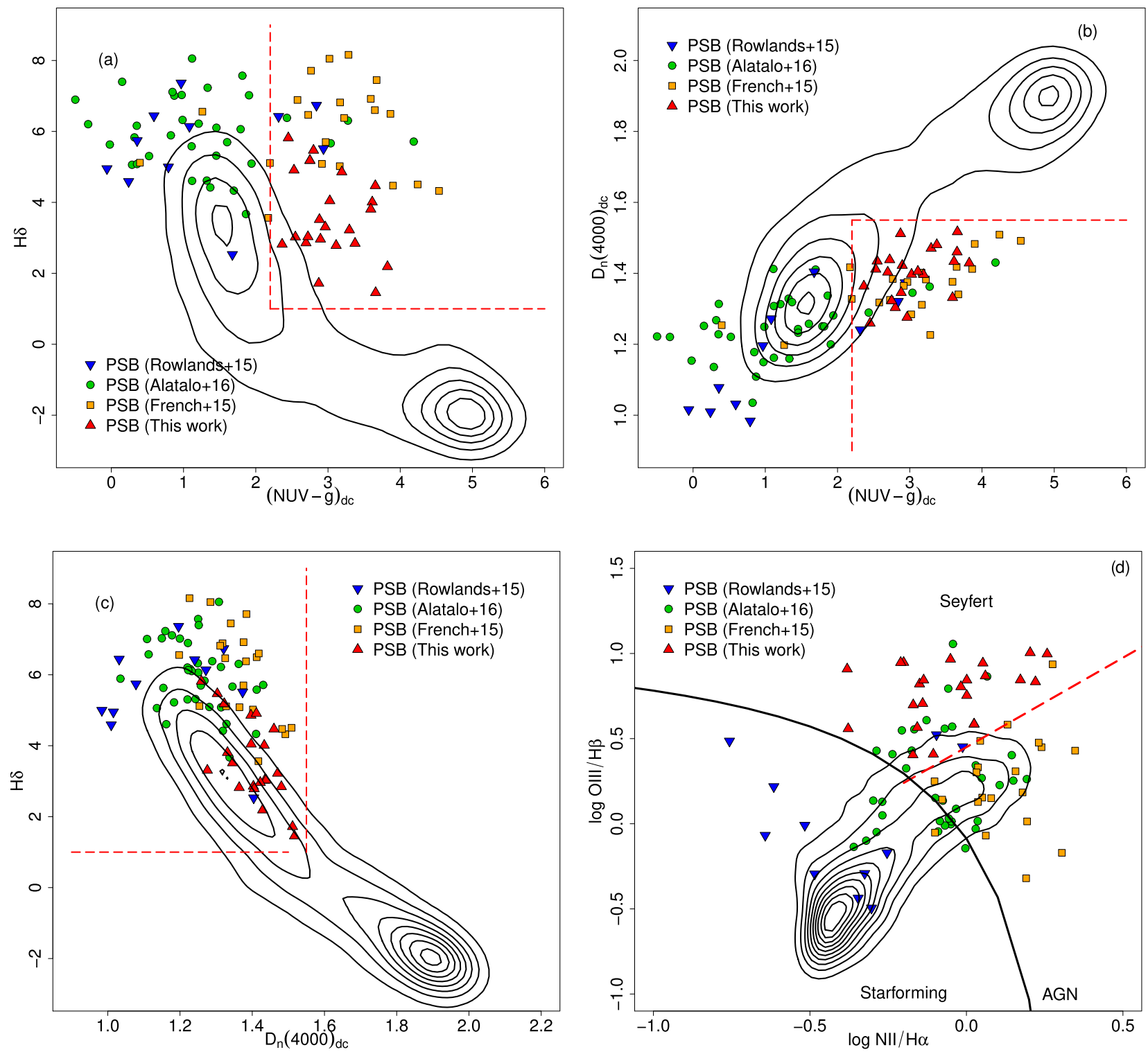

Figure 1. This figure shows the sample selection. The panels clearly show that PSB galaxies as a class differ strongly from normal SDSS galaxies and further that the various types of PSB of galaxies differ from one another depending on how the samples are selected. (a) H $\delta$ absorption equivalent width against dust-corrected NUV $-g$ colour. (b) The $D_{n}(4000)$ index versus the dust-corrected NUV $-g$ colour. (c) The H $\delta$ absorption equivalent width against the $D_{n}(4000)$ index. (d) The BPT emission line ratio AGN diagnostic. Seyferts are located in the upper corner of the AGN region (Kewley et al. 2001; Schawinski et al. 2007). The contours in all panels represent the number densities of SDSS galaxies at $z=0.02-0.06$ and with stellar mass $M=10^{10}$ and $10^{11} \mathrm{M}_{\odot}$. The coloured points are PSB galaxies. As detailed in Yesuf et al. (2014), the combination NUV $-g$ colour with $\mathrm{H} \delta$ and $D_{n}(4000)$ is useful in identifying late-stage PSB galaxies. The red triangles are the new sample of green-valley Seyfert PSBs selected in this work. They are selected quantitatively as objects that are $2 \sigma$ outliers from the loci of normal galaxies, at a given $D_{n}(4000)$, in their dust-corrected NUV $-g$ colours or H $\delta$ (see also fig. 4 of Yesuf et al. 2014). The red dashed lines outline the sample selection for the new Seyfert PSBs. The orange squares are PSBs studied by French et al. (2015). The green circles and the blue triangle are PSBs studied by Alatalo et al. (2016) and Rowlands et al. (2015), respectively.

sample selection of Yesuf et al. (2014). Most (19/22) of the galaxies in our sample have, within the measurement errors, $\mathrm{H} \delta$ absorptions $\gtrsim 3 \AA$, which is expected in PSBs with weak or strong bursts or rapidly truncated star-forming galaxies (e.g. Poggianti et al. 1999). Our sample on average has lower $\mathrm{H} \delta$ than do the aforementioned previous samples. Our PSBs are consistent with being later stage PSBs or PSBs with weaker starbursts compared to most of the PSBs in the other samples considered here. Note that the $\mathrm{H} \delta$ equivalent width is $\sim 2 \AA$ at $1 \mathrm{Gyr}$ after a starburst with star formation timescale $\tau=0.1 \mathrm{Gyr}$ and burst mass fraction $b_{\mathrm{f}}=20$ per cent. For starburst with $\tau=0.1 \mathrm{Gyr}$ and $b_{\mathrm{f}}=3$ per cent, $\mathrm{H} \delta \sim 0$ at $1 \mathrm{Gyr}$ (see Fig. A1 in Appendix A). Thus, not imposing $\mathrm{H} \delta>4 \AA$ cut helps select old PSBs. However, it remains uncertain whether these galaxies underwent weak or strong starbursts or rapidly truncated star formation.

It should be noted that at $z<0.06$, the SDSS fibre covers only $\sim 3.5 \mathrm{kpc}$ of the central region of a galaxy, and the spectroscopic measurements may not reflect the galaxy-wide values. On the other hand, the NUV - $g$ colour is an integrated galaxywide quantity. By combining the spectroscopic measurements with 
NUV $-g$ colour, we select only (PSB) galaxies that are fading galaxy wide.

\subsection{SMT CO observations}

The observations were carried out using the Arizona Radio Observatory SMT on Mount Graham, Arizona. The observing runs were performed in 2015 February 25-March 10 and in 2016 March 425. We follow the same instrument set-up and observing strategy as French et al. (2015). Namely, we used the $1 \mathrm{~mm}$ Atacama Large Millimeter Array (ALMA) Band 6 dual polarization sideband separating SIS (superconductor-insulator-superconductor) receiver and $1 \mathrm{mHz}$ filterbank to measure the $\mathrm{CO}(2-1) 230.5 \mathrm{GHz}$ emission line. The beam size of the SMT for this line is about 33 arcsec. Beam switching was done with the secondary at $2.5 \mathrm{~Hz}$ switching rate and a throw of 120 arcsec, in the BSP (beam switching plus position switching) mode. Calibration using a hot load and the standard chopper wheel method was performed every $6 \mathrm{~min}$. Calibration using a cold load was performed at every tuning. The observing times range between 4 and $9 \mathrm{~h}$. French et al. (2015) observed a subset of 13 PSBs using both SMT and IRAM, which we use to guide comparisons between SMT- and IRAM-observed samples.

The data reduction is done using CLASS, a program within the GILDAS software package. ${ }^{2}$ The main beam efficiency is calculated using Jupiter in each polarization. A first-order polynomial baseline is subtracted from the spectra using data between $[-600,600] \mathrm{km}$ $\mathrm{s}^{-1}$, excluding the central regions of $[-300,300] \mathrm{km} \mathrm{s}^{-1}$. The spectra are scaled using the main beam efficiency, and are co-added by weighting with the root-mean-square (rms) noise. The spectra are rebinned to $14 \mathrm{~km} \mathrm{~s}^{-1}$ velocity bins. The typical rms error per bin is $1-2 \mathrm{mK}$. Thus, we achieve similar sensitivity as previous works (Saintonge et al. 2011; French et al. 2015; Rowlands et al. 2015; Alatalo et al. 2016).

To calculate the integrated $\mathrm{CO}$ line intensity, $I_{\mathrm{CO}}$, we fit a Gaussian profile to each line, allowing the peak velocity to differ from the optical systemic velocity by up to $200 \mathrm{~km} \mathrm{~s}^{-1}$. The statistical uncertainty in the line intensity is calculated following Young et al. (2011) as $\sigma_{\mathrm{I}}=(\Delta v)^{2} \sigma^{2} N_{\mathrm{I}}\left(1+\frac{N_{\mathrm{I}}}{N_{\mathrm{b}}}\right)$, where $\Delta v$ is the channel velocity width, $\sigma$ is the channel rms noise, $N_{\mathrm{I}}$ is the number of channels used to integrate over the line and $N_{\mathrm{b}}$ is the number of channels used to fit the baseline. When the line is not detected, the upper limits of the line intensity are calculated as three times the statistical uncertainty. Following Solomon et al. (1997), the CO line luminosity in $\mathrm{K} \mathrm{km} \mathrm{s}^{-1} \mathrm{pc}^{2}$ is $L_{\mathrm{CO}}^{\prime}=23.5 \Omega_{s * b} D_{\mathrm{L}}^{2} I_{\mathrm{CO}}(1+z)^{-3}$, where $\Omega_{s^{\star} b}$ is the solid angle of the source convolved with the beam, $z$ is the redshift from the SDSS optical spectrum and $D_{\mathrm{L}}$ is the luminosity distance in Mpc. If the source is much smaller than the beam, then $\Omega_{s^{\star} b} \approx$ $\Omega_{b}$. We do not know the CO emitting sizes of our galaxies. We adopt this approximation as a simplifying assumption. We do not expect this to affect our conclusion. Previous works also adopt the same approximation. The fact that SMT has larger beam size than IRAM, and $\mathrm{CO}(2-1)$ is known to be more centrally concentrated than CO (1-0), makes the effect of this approximation less significant in this work compared to previous works. French et al. (2015) estimated for their sample that the $L_{\mathrm{CO}}^{\prime}$ may be underestimated by about $1.5 \times$ because of this effect.

The molecular gas mass can be calculated from $L_{\mathrm{CO}}^{\prime}$ by assuming a CO conversion factor (mass-to-light ratio) $\alpha_{\mathrm{CO}}, M\left(\mathrm{H}_{2}\right)=\alpha_{\mathrm{CO}} L_{\mathrm{CO}}^{\prime}$. We assume an $\alpha_{\mathrm{CO}}=4.3 \mathrm{M}_{\odot}\left(\mathrm{K} \mathrm{km} \mathrm{s}^{-1} \mathrm{pc}^{2}\right)^{-1}$ in Milky Way disc

\footnotetext{
${ }^{2}$ http://www.iram.fr/IRAMFR/GILDAS/
}

(Bolatto, Wolfire \& Leroy 2013) and $R_{21}=L_{\mathrm{CO}(2-1)}^{\prime} / L_{\mathrm{CO}(1-0)}^{\prime}=1$. As discussed in Section 5.3, choosing a lower value of $\alpha_{\mathrm{CO}}$ strengthens our main conclusion. The ratio $R_{21}$ is uncertain (Leroy et al. 2013; Sandstrom et al. 2013). Leroy et al. (2013) found $R_{21} \sim 0.7$ \pm 0.3 in nearby disc galaxies. We adopt the Galactic $\alpha_{\mathrm{CO}}$ value throughout the paper unless explicitly stated, and all previous measurements are adjusted to our assumed value of $\alpha_{\mathrm{CO}}$. The observations are summarized in Table 1, the co-added spectra are given in Figs 2 and 3 and the SDSS cut-out images of the Seyfert PSBs are shown in Fig. 4.

\section{OVERVIEW OF STATISTICAL METHODS}

In this section, we briefly review the statistical methods and tests used in the next section. Readers interested in more details of the methods should refer to the references provided.

In this section, we are concerned with how to analyse data that include both $\mathrm{CO}$ detections and $\mathrm{CO}$ upper limits, and make inference that is consistent with the incomplete information at hand. In statistics, a data set is called censored when the value of a measurement is only partially known to be above or below a threshold. Survival analysis is an area of statistics that mostly deals with modelling censored data such as time to an event (e.g. Feigelson \& Nelson 1985; Klein \& Moeschberger 2005; Halsel 2012). We use existing survival analysis methods to properly extract information from our censored CO observations.

Let $T$ denote a positive random variable representing time to an event of interest, say death. The survival function is the probability that an individual survives beyond time $t, S(t)=\operatorname{Pr}(T \geq$ $t)=1-F(t)$, where $F(t)$ is the cumulative distribution function. The Kaplan-Meier estimator (Kaplan \& Meier 1958) is a nonparametric maximum likelihood estimator of the survival function, even in the presence of censoring. A non-parametric method does not require an assumption that the data follow a specific probability distribution. Instead, the data are ranked from smallest to largest, providing information on the relative positions of each observation. In the case of ties, Kaplan-Meier method assigns the smallest rank to each observation. The censored observations are also used in calculating the ranks. Let there be a set of $\left\{t_{k}\right\}_{k=1}^{n}$ data points and of these let $t_{1}^{\prime}<t_{2}^{\prime}<t_{3}^{\prime}, \ldots, t_{r}^{\prime}$ be ranked, distinct uncensored values. At each time point $t_{j}^{\prime}$, we observe $d_{j}$, the number of deaths, $c_{j}$, the number of censored observations between the time $t_{j}^{\prime}$ and $t_{j-1}^{\prime}$ and, $n_{j}$, the number of individuals at risk just prior to the time $t_{j}^{\prime}$. In other words, $n_{j}$ is the total sample size minus those who are censored or have died before $t_{j}^{\prime}, n_{j}=n-c_{j}-d_{j}=\sum_{l \geq j}\left(c_{l}+d_{l}\right)$. The Kaplan-Meier estimator has a form: $\hat{S}(t)=\prod_{j: t_{j}^{\prime}<t}\left(1-d_{j} / n_{j}\right)$. It is a step function with jumps at times $t_{j}^{\prime}$.

To compute the empirical cumulative distribution function (ECDF), using the Kaplan-Meier method, we use the NADA package in R programming language (Halsel 2012; Lee \& Lee 2015). We use the function cenfit in the NADA package to compute the Kaplan-Meier estimator for the CO data (Halsel 2012; Lee \& Lee 2015).

The survival curves or ECDF of two groups can be compared using the log-rank test, which tests the null hypothesis that two groups have the same distribution against the alternative hypothesis that two groups have different distributions (Mantel 1966; Cox 1972; Harrington \& Fleming 1982; Martinez 2007). If the data are uncensored, the log-rank test gives similar result to the MannWhitney test. We use the routine cendiff in the NADA package to do the log-rank test. 
Table 1. Summary of the CO observations of the new Seyfert transition PSBs. The column names are, from left to right, target name, right ascension, declination, redshift, $4000 \AA$ A break, dust-corrected NUV - $g$ colour, H $\delta$ absorption equivalent width, WISE flux ratio, stellar mass, molecular gas mass and detection flag. For the non-detections, the given molecular masses are $3 \sigma$ upper limits.

\begin{tabular}{|c|c|c|c|c|c|c|c|c|c|c|}
\hline Target & RA & Dec. & $z$ & $D_{n}(4000)_{\mathrm{dc}}$ & $(\mathrm{NUV}-g)_{\mathrm{dc}}$ & $\mathrm{H} \delta$ & $\log f_{12} / f_{4.6}$ & $\log M_{\star} / \mathrm{M}_{\odot}$ & $\log M_{\mathrm{H}_{2}} / \mathrm{M}_{\odot}$ & $\log M_{\mathrm{H}_{2}} / M_{\star}$ \\
\hline TPSB2 & 134.61915 & 0.02347 & 0.0285 & 1.32 & 2.8 & 5.2 & 0.59 & 10.40 & 8.64 & -1.76 \\
\hline TPSB5 & 173.41286 & 52.67458 & 0.0490 & 1.40 & 3.2 & 4.9 & 0.22 & 10.19 & $<9.10$ & $<-1.09$ \\
\hline TPSB6 & 170.94591 & 35.44231 & 0.0341 & 1.42 & 2.5 & 4.9 & 0.52 & 10.29 & $<8.97$ & $<-1.32$ \\
\hline TPSB7 & 203.56173 & 34.19415 & 0.0236 & 1.31 & 2.8 & 5.5 & 0.44 & 10.15 & 8.62 & -1.53 \\
\hline TPSB8 & 189.51736 & 48.34506 & 0.0306 & 1.44 & 3.6 & 4.0 & 0.04 & 10.21 & $<8.81$ & $<-1.40$ \\
\hline TPSB10b & 126.01534 & 51.90432 & 0.0315 & 1.36 & 2.4 & 2.8 & 0.72 & 10.12 & $<9.12$ & $<-1.00$ \\
\hline TPSB11 & 137.87485 & 45.46828 & 0.0268 & 1.43 & 2.6 & 3.0 & 0.64 & 10.70 & 8.93 & -1.77 \\
\hline TPSB12 & 139.49938 & 50.00218 & 0.0342 & 1.41 & 3.1 & 0.8 & 0.53 & 10.33 & $<8.94$ & $<-1.39$ \\
\hline TPSB13 & 173.16774 & 52.95040 & 0.0266 & 1.50 & 1.8 & 2.4 & 0.48 & 10.54 & $<8.68$ & $<-1.86$ \\
\hline TPSB14 & 178.62255 & 42.98021 & 0.0235 & 1.51 & 2.9 & 1.7 & 0.57 & 10.05 & $<8.71$ & $<-1.34$ \\
\hline TPSB15 & 179.02851 & 59.42492 & 0.0320 & 1.52 & 3.7 & 1.5 & 0.41 & 10.49 & $<8.85$ & $<-1.64$ \\
\hline TPSB20 & 240.65806 & 41.29344 & 0.0348 & 1.45 & 2.7 & 3.0 & 0.08 & 10.59 & $<8.97$ & $<-1.62$ \\
\hline TPSB21 & 247.63604 & 39.38420 & 0.0305 & 1.42 & 2.9 & 3.0 & 0.55 & 10.54 & $<8.82$ & $<-1.72$ \\
\hline TPSB24 & 145.18542 & 21.23427 & 0.0244 & 1.43 & 3.8 & 2.2 & 0.71 & 10.33 & $<8.60$ & $<-1.73$ \\
\hline TPSB26 & 172.08298 & 27.62209 & 0.0321 & 1.42 & 3.1 & 2.8 & 0.18 & 10.31 & $<8.89$ & $<-1.42$ \\
\hline TPSB28 & 222.65772 & 22.73433 & 0.0210 & 1.33 & 3.6 & 3.8 & 0.60 & 10.09 & $<8.47$ & $<-1.62$ \\
\hline
\end{tabular}

The Kendall's $\tau$ is a non-parametric correlation coefficient that can be used for testing trends in both censored and uncensored data (e.g. Halsel 2012). $\tau$ ranges between -1 and 1. $\tau=0$ is no correlation, $\tau=1$ is a perfect correlation and $\tau=-1$ is a perfect anti-correlation.

In the uncensored case, the slope of a linear regression can be estimated using the Theil-Sen slope (Sen 1968), which is the median of all pairwise slopes between two data points. This slope results in the Kendall's $\tau$ of 0 for the correlation between the residuals and the covariate, $X$. For censored data, the slope can be estimated using the extended version called Akritas-Theil-Sen estimator (Akritas, Murphy \& LaValley 1995). The intercept of the linear regression is the median of the residual. We use the cenken routine in NADA package to compute the Kendall's $\tau$ correlation coefficient and fit the ATS regression line to our censored CO data (Halsel 2012; Lee \& Lee 2015).

\section{RESULTS}

Fig. 5 compares the ECDFs of $\log f_{\text {gas }}$ of normal star-forming galaxies $\left(D_{n}(4000)<1.6\right)$ with that of PSBs while Fig. 6 compares the ECDF of the star-forming galaxies with Seyfert PSBs. The vertical probability axis in both plots tracks the estimated KaplanMeier percentiles of galaxies with gas fraction less than a given threshold (Kaplan \& Meier 1958; Halsel 2012). The $\log f_{\text {gas }}$ curves for the PSBs and non-PSBs are similar at high gas fractions but are different at lower gas fractions. The log-rank test indicates that ECDFs of all PSBs or Seyfert-only PSBs are significantly different from that of the young non-PSBs. When the ECDF for Seyfert PSBs is compared to that of the young non-PSBs, the test gives a $\chi^{2}$ of 24 on 1 degree of freedom corresponding to a $p$-value of $10^{-6}$. Therefore, the null hypothesis that the EDCFs for these two populations are the same can be rejected at a $4.8 \sigma$ significance level. Similarly, the test gives a $\chi^{2}$ of 11.4 on 1 degree of freedom, corresponding to a $p$-value of $7 \times 10^{-4}$, when EDCFs of all PSBs and non-PSBs with $D_{n}(4000)<1.6$ are compared.

Having established that there is a statistically significant difference between the gas fraction of young star-forming non-PSB galaxies and of PSBs in general, and of Seyfert PSBs in particular, in the rest of this section we explore in more detail where these trends come from. We first demonstrate that the WISE flux ratio between 12 and $4.6 \mu \mathrm{m}$ is an excellent proxy for $\log f_{\text {gas }}$ using the CO Legacy Database (COLD) GASS sample of non-PSBs (Saintonge et al. 2011) and then use it as a tool to constrain the gas fraction evolution in PSBs. Stellar populations younger than $0.6 \mathrm{Gyr}$ dominate the $12 \mu \mathrm{m}$ emission, and this ratio is known to correlate well with the specific SFR (sSFR; Donoso et al. 2012). The AGN hot dust emission is mostly between $\sim 3$ and $5 \mu \mathrm{m}$; some have suggested that this ratio is not appreciably affected by AGN emission and that it can trace the sSFR in AGN (Donley et al. 2012; Donoso et al. 2012). Yesuf et al. (2014) have shown that starbursts and PSBs form a time sequence in this ratio, with starbursts having the highest ratios and quiescent PSBs having ratios similar to those of green-valley and red-sequence galaxies.

Fig. 7 shows the WISE flux ratio $\log f_{12} / f_{4.6}$ versus $\log f_{\text {gas }}$ for all normal galaxies in the COLD GASS survey. As shown in Fig. A3 of Appendix A, the general population of SDSS galaxies show bimodal $\log f_{12} / f_{4.6}$ distribution that correlates with $D_{n}(4000)$. Star-forming galaxies have ratios between $\log f_{12} / f_{4.6} \sim 0.2$ and 1 while quiescent galaxies have ratios between $\log f_{12} / f_{4.6} \sim-0.8$ and 0.0 . A tight correlation is observed between $\log f_{12} / f_{4.6}$ and $\log f_{\text {gas }}$ in the COLD GASS sample, indicating an evolutionary process in normal galaxies in which sSFR declines as gas is used up. The slope of the bestfitting line is $\beta=1.13$ and its intercept is $\beta_{0}=-1.8$. The Kendall's $\tau$ correlation coefficient for the fit is 0.64 . The statistical significance of the correlation is more than $5 \sigma$. One can easily show that $\beta$ and $\beta_{0}$ are related to the logarithm of the molecular gas depletion time 

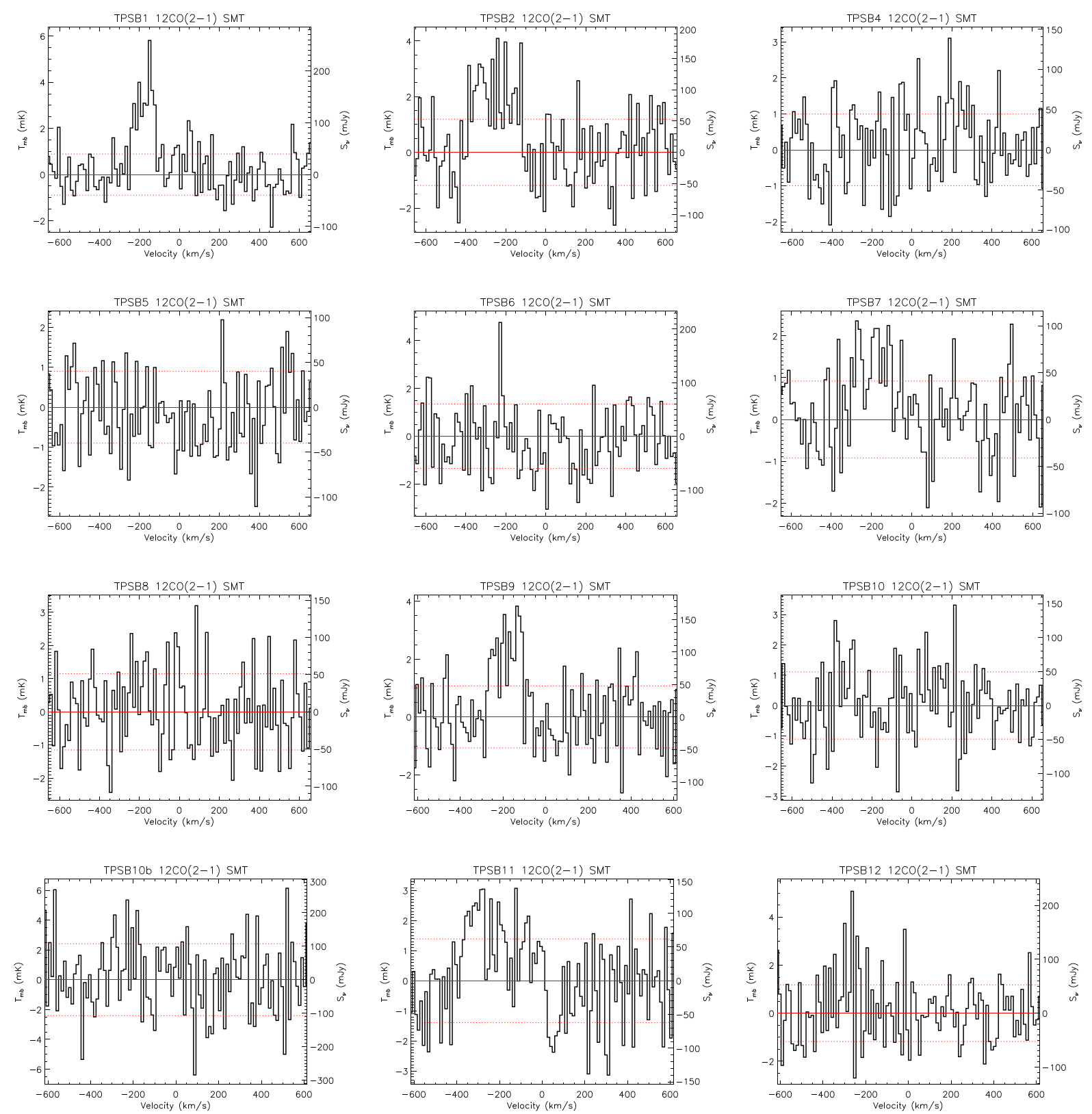

Figure 2. SMT CO (2-1) spectra for Seyfert PSBs. Spectra are shown in units of both main beam temperature $T_{\mathrm{mb}}(\mathrm{mK})$ and $S_{v}$ (Jy). The dashed red lines represent the rms of the binned data. The data are binned by $14 \mathrm{~km} \mathrm{~s}^{-1}$.

at a given sSFR by the relation: $\log \tau_{\text {dep }}=\hat{\beta} \log \mathrm{sSFR}+\hat{\beta}_{0}$, where $\hat{\beta}=(\beta / \alpha-1), \hat{\beta_{0}}=-\beta\left(\alpha_{0} / \alpha+0.769\right)+\beta_{0}$, where $\alpha$ and $\alpha_{0}$ define the relation: $\log \mathrm{SSFR}=\alpha\left(\log f_{12} / f_{4.6}+0.769\right)+\alpha_{0}$. Donoso et al. (2012) computed the relation between sSFR and 4.6-12 $\mu \mathrm{m}$ colour in Vega magnitude and 0.769 is subtracted to change the colour to $\mathrm{AB}$ magnitude. They found $\alpha=2.5 \times 0.775=1.938$ and $\alpha_{0}=-12.56$ for the SDSS star-forming galaxies. Using these values with our fitted values of $\beta$ and $\beta_{0}$ gives $\hat{\beta}=-0.42$ and $\hat{\beta}_{0}=4$.7. In comparison, Huang \& Kauffmann (2014) found $\hat{\beta}=-0.37 \pm 0.04$ and $\hat{\beta}_{0}=5.45 \pm 0.42$ for the COLD GASS sample. The authors used the GALEX FUV and WISE $22 \mu \mathrm{m}$ to estimate the sSFR. Our inferred depletion times are consistent with Huang \& Kauffmann (2014) but there is likely a systematic offset in the two SFR estimates.
Fig. 8 shows the relationship between the WISE flux ratio $\log f_{12} / f_{4.6}$ and $\log f_{\text {gas }}$ for PSBs. They also show statistically significant $(5 \sigma)$ correlation between these two quantities. The slope of the best-fitting line is $\beta=1.46$ and its intercept is -2.1 . Collectively, the broad overall trend suggests same correlation is at work: lower molecular gas fractions mean lower sSFR or dust heating. But the scatter is much larger, and looking in detail we can see that certain subpopulations of PSBs statistically differ. A major insight from this figure is that the sSFR histories of the PSBs and of Seyferts PSBs may not be as simple or well behaved as normal galaxies. Hence, the use of standard age indicators may lead to different results depending on indicator used. This topic will be explored in future papers. The reason for the low $\log f_{\text {gas }}$ of Seyferts in Fig. 6 is consistent with low sSFR upper limits inferred from $\log f_{12} / f_{4.6}$. 

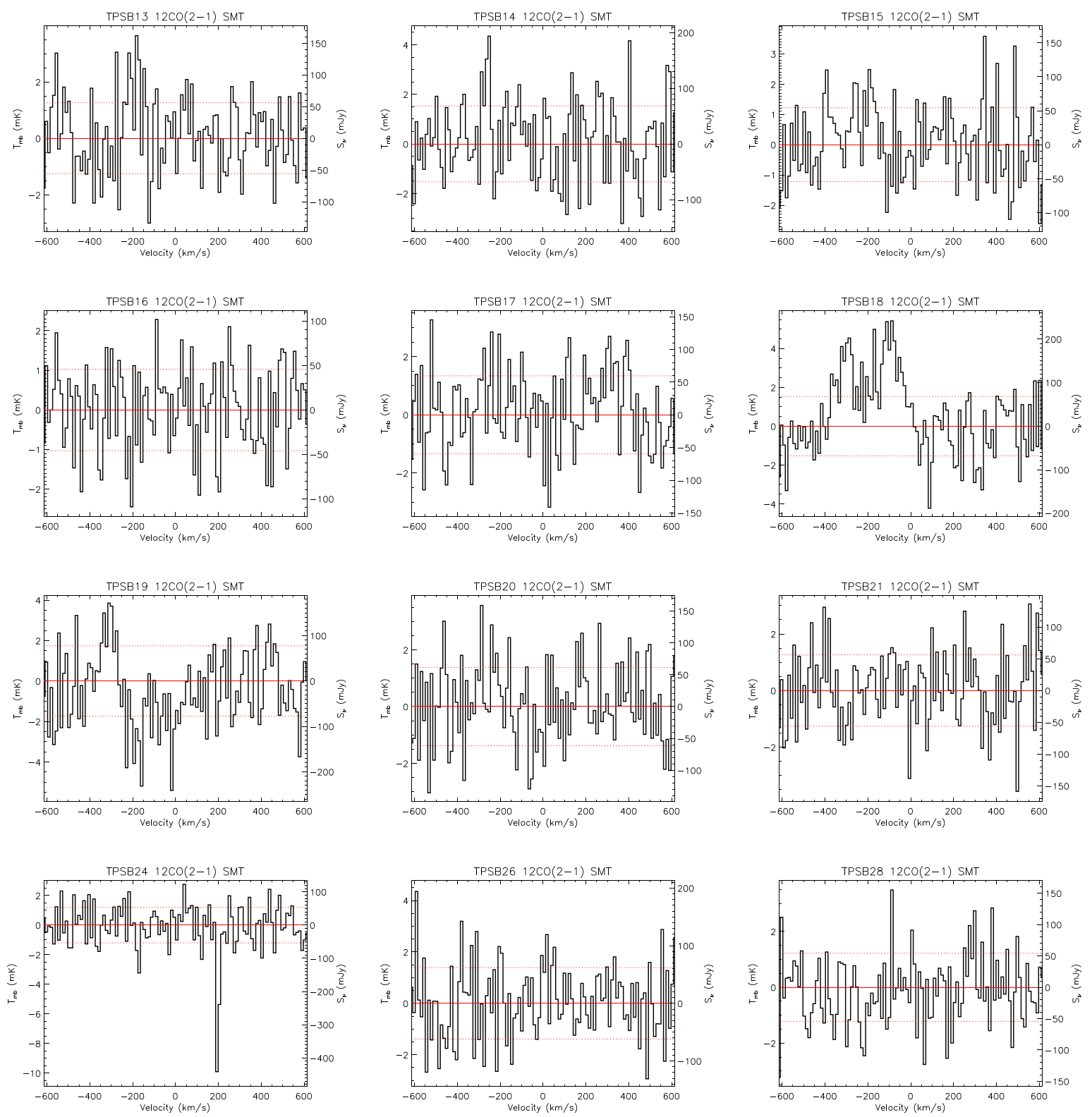

Figure 3. $\mathrm{CO}$ (2-1) spectra (continuation of Fig. 2).

Table 2 summarizes the distribution of molecular gas fraction of our sample and of the previous samples of PSBs. Our sample has a $\log f_{\text {gas }}$ distribution with mean of 0.025 and dispersion 0.018. 95 per cent of our PSBs have gas fractions below 0.07 while 50 per cent have gas fractions below 0.02 . The previous samples have means and dispersions of $\sim 0.1-0.2$. It is particularly interesting that the French et al. (2015) sample, which may also contain late-stage PSBs, has $\log f_{\text {gas }}$ distribution with mean of 0.08 and dispersion 0.12 . The majority of PSBs from Rowlands et al. (2015) and Alatalo et al. (2016) samples have both high $\log f_{12} / f_{4.6}$ and high molecular gas fractions in comparison to our sample. The PSBs in these two works may be precursors to the PSBs in our sample or to those in the French et al. (2015) sample.

\section{DISCUSSION}

In this section, we discuss in detail how gas fractions in our sample compare with those observed in previous samples of PSBs. We will also discuss the general implication of our result and the uncertainty in the hitherto assumed $\mathrm{CO}$ conversion factor.

\subsection{Comparison of our sample with the previous samples of PSBs}

We study the molecular gas evolution of 116 PSB galaxies, of which 22 have new observations undertaken by us while the rest are compiled from the literature (French et al. 2015; Rowlands et al. 2015; Alatalo et al. 2016). The galaxies in the new sample are selected using their spectral indices and NUV $-g$ colours, to identify them as green-valley PSBs, and their emission line ratios, to identify them as Seyferts. Our sample represents about two-thirds of the Seyferts PSBs in the combined sample. The Seyfert PSBs from the previous works were serendipitously selected and are mostly in the blue cloud. We show that $\log f_{\text {gas }}$ distribution of the Seyfert PSBs and that of PSBs in general are significantly different from that of normal star-forming galaxies. The molecular gas fraction in the 


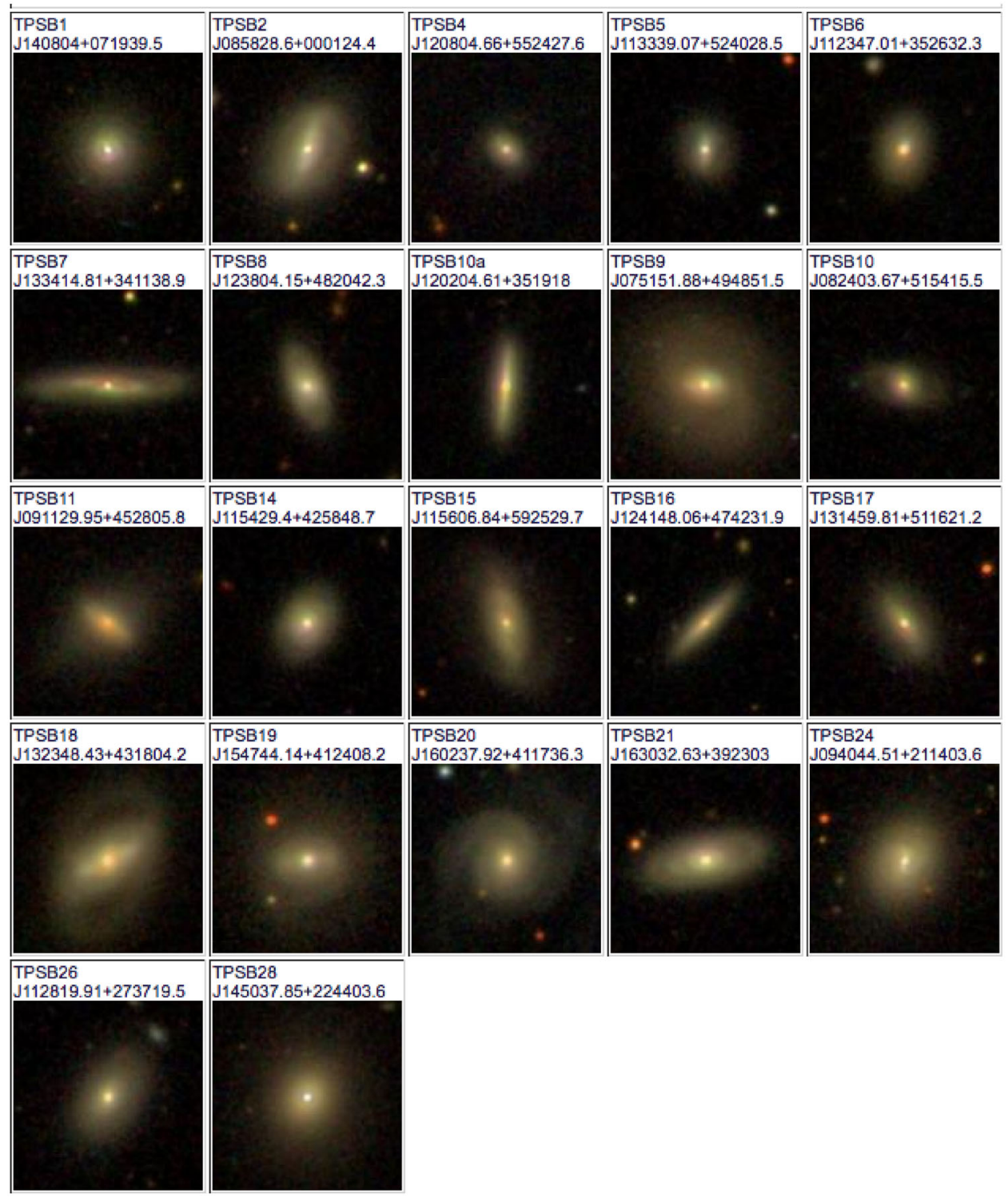

Figure 4. SDSS cut-out images of the green-valley Seyfert PSBs. They show prominent bulges. But they do not show strong merger signatures and dust extinctions, unlike some of the previously studied PSBs (e.g. Alatalo et al. 2016). This, together with their older stellar populations and red NUV-optical colours, indicates that they are late-stage PSBs.

Seyfert PSBs decreases with the WISE ratios $\log f_{12} / f_{4.6}$. If there is an evolutionary sequence in Fig. 8, from upper right to lower left, it would be plausible to conclude that our new Seyferts are fairly aged galaxies located nearing the middle of the gas-exhaustion cycle. This conclusion would be consistent with our selection of these galaxies based on integrated UV colour as members of the green valley and well on their way to gas exhaustion. The systematically lower gas content of the new Seyferts relative to the PSBs in Fig. 8 might then be interpreted as the action of late AGNs in them in finally clearing out the gas.

However, we call attention to an important puzzle. In Fig. 8, the French et al. (2015) sample as a whole shows a significant correlation between $\log f_{12} / f_{4.6}$ and $\log f_{\text {gas }}$; the PSBs with high $\log f_{12} / f_{4.6}$ have high gas fractions. This is puzzling because the French et al. (2015) galaxies were selected to have very low $\mathrm{H} \alpha$, which is the gold standard for SFR. Thus, the $\log f_{12} / f_{4.6}$ and $\mathrm{H} \alpha$ as SFR indicators disagree for the subset of the French et al.
(2015) sample with high $\log f_{12} / f_{4.6}$. However, A-star heating is significant during the PSB phase, which can artificially boost IRbased SFR indicators (Hayward et al. 2014; Utomo et al. 2014). An analysis of multiple SFR indicators, including extinction-free $1.4 \mathrm{GHz}$ measurements in section 2.5 of French et al. (2015), shows these galaxies are unlikely to have significant dust obscuration. Analyses of the near- and far-IR properties of this sample are the subject of a forthcoming study (Smercina et al., in preparation).

The episodic lifetime of an individual AGN event is currently not well constrained, and it may range between $\sim 0.3$ and 1 times the effective AGN lifetime (Hopkins \& Hernquist 2009). The effective AGN lifetime for the Seyfert PSBs is likely to be $\sim 2$ Gyr. This estimate uses the median $\sigma=110 \mathrm{~km} \mathrm{~s}^{-1}$ to estimate the median black hole mass, $10^{7.4} \mathrm{M}_{\odot}$, from the $M_{\mathrm{BH}}-\sigma$ relation (Kormendy 2013), the median dust-corrected $\mathrm{O}$ III luminosity, $10^{7.3} \mathrm{~L} \odot$, to estimate the Eddington ratio, $\lambda=0.003$ (Lamastra et al. 2009), and fig. 9 of Hopkins \& Hernquist (2009) to estimate the effective AGN lifetime 


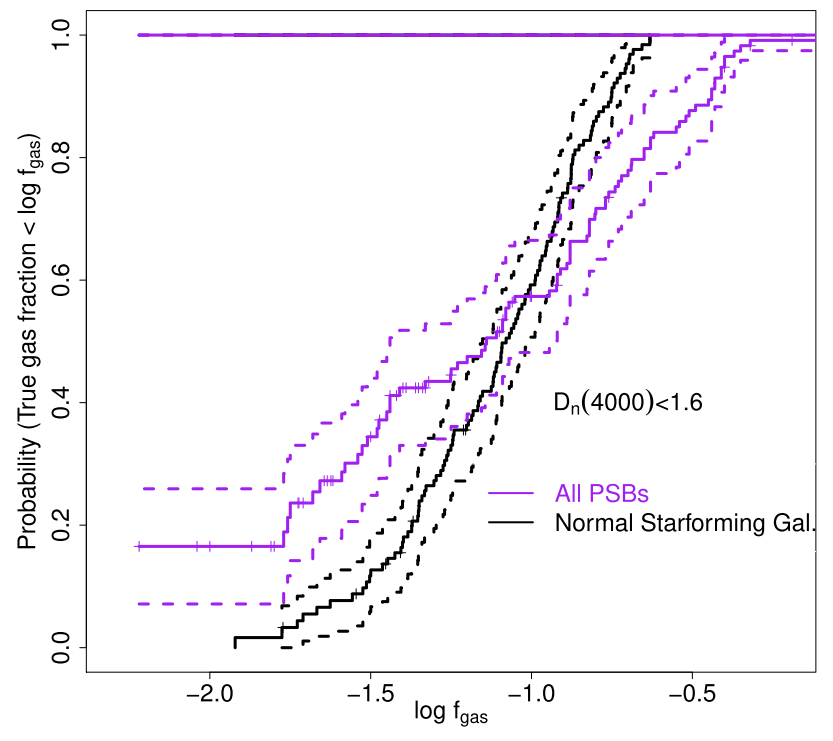

Figure 5. This figure compares the ECDF of molecular gas fraction, $\log f_{\text {gas }}$, of PSBs and of star-forming non-PSBs. The ECDF is estimated using the Kaplan \& Meier (1958) estimator and takes into account the upper limits, which are shown as $+\mathrm{s} .95$ per cent confidence curves are shown as dashed lines. The vertical axis denotes the estimated proportion of galaxies with gas fraction less than a given observed value. The distribution of gas fractions in PSBs is significantly broader (different) from that of non-PSBs. For example, the proportion of PSBs with gas fractions below $\sim 5$ percent is higher than that of star-forming non-PSB galaxies. See the text in Section 4 for a quantitative test that compares the two distributions.

from $M_{\mathrm{BH}}$ and $\lambda$. The effective AGN lifetime for the Seyfert PSBs is longer than the quenched PSB lifetime of $\sim 1 \mathrm{Gyr}$, which suggests that, given the above estimate of the episodic lifetime, the quenched PSBs may experience second episode of AGN activity or no AGN activity at all within their typical lifetime. Perhaps the variation in AGN activity time-scale is linked to a wide range in gas fraction observed in the French et al. (2015) sample, if these PSBs are truly quiescent galaxies, and AGN are linked to the removal of gas in the French et al. (2015) galaxies with $\operatorname{low} \log f_{12} / f_{4.6}$ and in our sample.

We used the $\log f_{12} / f_{4.6}$ ratio as a crude first-order indicator of star formation activity in both AGN and non-AGN galaxies. Some variation is expected between the ratios of the two classes (Donoso et al. 2012). It is not clear if these variations are due to additional AGN dust heating or preferentially aged stellar populations in AGN host galaxies. Understanding the details of this variation is not important for conclusions of the current work, but future studies of the effect of AGN contamination on $\log f_{12} / f_{4.6}$ will be useful.

Furthermore, it should be noted that the gas fractions in very nearby early-type galaxies are found to be $\lesssim 0.1$ per cent (e.g. Young et al. 2011). Many of the PSBs considered here have molecular gas fractions already consistent with detections in these early-type galaxies. A full comparison with early-type galaxies is complicated by the lower redshifts and smaller physical apertures of the Young et al. (2011, ATLAS 3D) sample. Deeper observations of the nondetected PSBs will be necessary to study the full range in their molecular gas properties, and to compare to the deep observations of the ATLAS 3D sample of early-type galaxies.
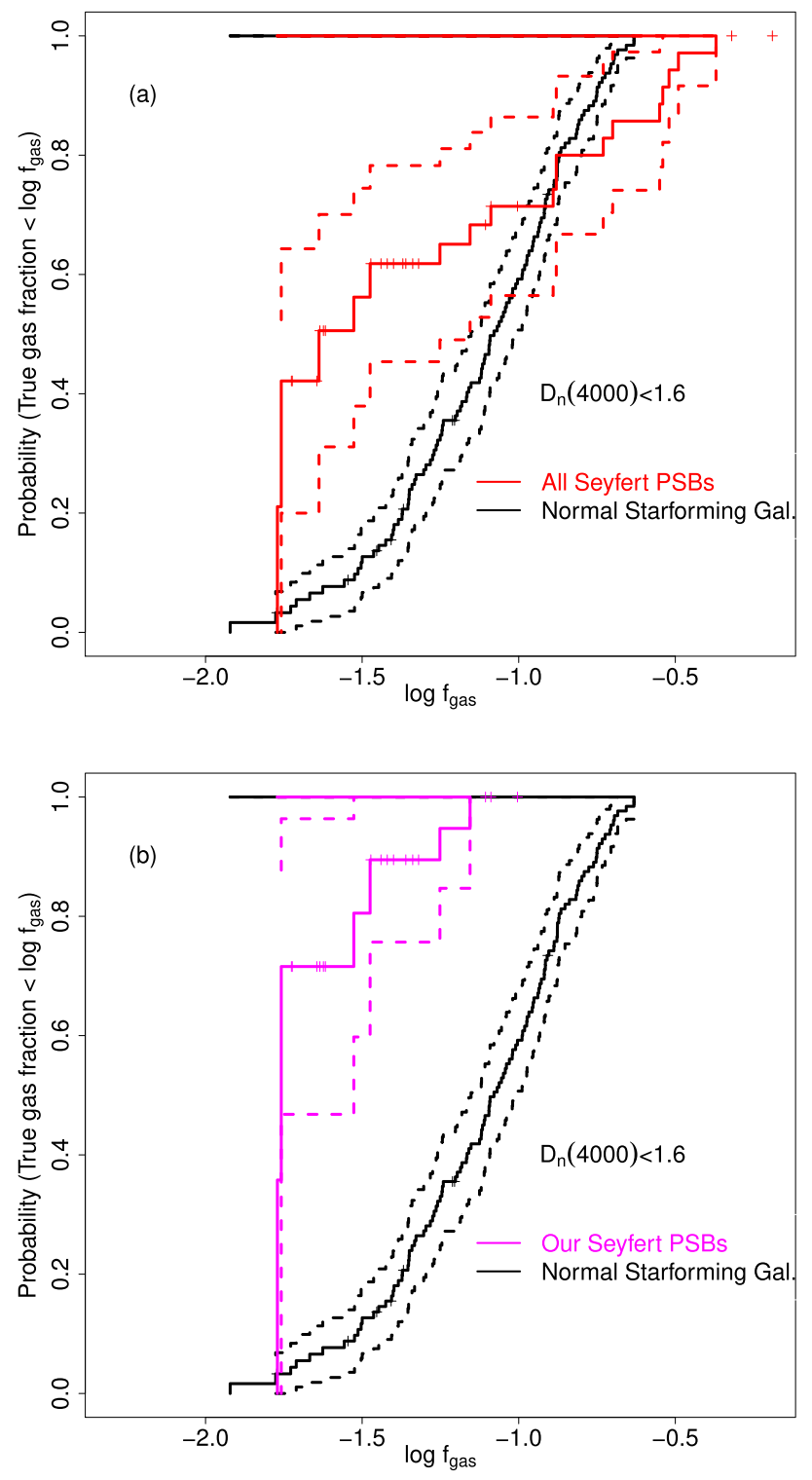

Figure 6. Panel (a) is similar to Fig. 5 but compares the ECDF of molecular gas fraction, $\log f_{\text {gas }}$, of all Seyfert PSBs and of star-forming non-PSBs. The distribution of gas fractions of Seyfert PSBs is significantly broader (different) than that of non-PSBs of similar $D_{n}(4000)$. A higher proportion of Seyfert PSBs have gas fraction fractions below $\sim 10$ per cent. Panel (b) only plots our new green-valley Seyfert PSB sample.

\subsection{Implication for galaxy evolution}

It is thought that star formation quenching happens in both slow and fast modes (e.g. Faber et al. 2007; Cheung et al. 2012; Fang et al. 2012; Barro et al. 2013; Dekel \& Burkert 2014; Schawinski et al. 2014; Yesuf et al. 2014; Woo et al. 2015; Pandya et al. 2016). The existence of these modes, their relative importance and the physical mechanisms that drive them are still open questions in galaxy evolution. Some works have indicated that PSBs quench rapidly and may exemplify an important mode quenching for the build-up of the red sequence (Kaviraj et al. 2007; Wild et al. 2009, 2016; Wong et al. 2012; Yesuf et al. 2014), while others have questioned their importance (De Lucia et al. 2009; Dressler et al. 2013). 


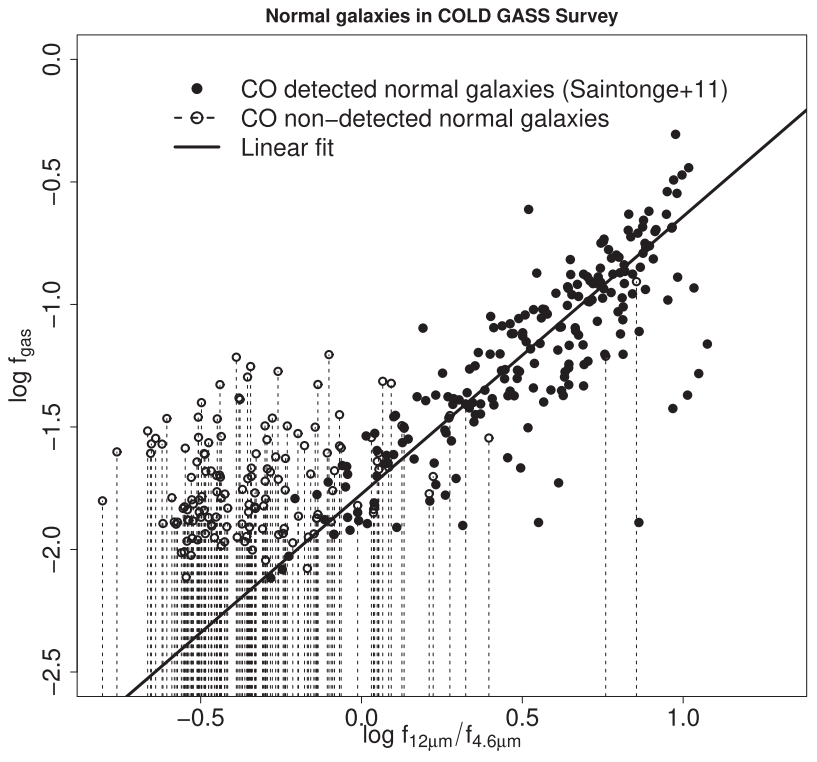

Figure 7. WISE flux ratio $\log f_{12} / f_{4.6}$ versus molecular gas fraction for all galaxies in COLD GASS survey (Saintonge et al. 2011). Young starforming galaxies have high $\log f_{12} / f_{4.6}$ while old quiescent galaxies have low $\log f_{12} / f_{4.6}$. The filled circles denote galaxies with $\mathrm{CO}$ detections while the open circles and the dashed lines denote non-detected galaxies and their upper limits. The black line is the linear fit to the data and it includes the upper limits. The $\log f_{12} / f_{4.6}$ is an excellent proxy for the molecular gas fraction.

Recent studies found large gas fractions in PSBs complicating the picture of their rapid evolution to the red sequence (French et al. 2015; Rowlands et al. 2015; Alatalo et al. 2016). However, these works have been biased against finding PSBs with Seyfertlike emission lines in the UV-optical green valley. In this work, we have observed the molecular gas properties of 22 such galaxies. The combined data presented in this work cannot rule out AGN feedback, and as a whole are consistent with a removal or destruction of molecular gas at later stages by AGN feedback or no AGN feedback at all if different subsamples of PSBs are not evolutionary connected. Better and deeper observations, especially at a later stage of PSB evolution, are needed to firmly and more directly test the merger-induced AGN feedback hypothesis. In the theoretical front, developments in cosmological simulations with AGN feedback are needed to be compared to these future observations.

\subsection{The effect of $\mathrm{CO}$ conversion factor}

The CO luminosity to the molecular hydrogen conversion factor is a widely acknowledged source of uncertainty in studies that use $\mathrm{CO}$ as a tracer of molecular hydrogen (Bolatto et al. 2013). Up to this point, we have assumed that all objects including PSBs have a conversion factor similar to the factor observed in the Milky Way disc. There is no study to date that measured the conversion factor in PSBs. As discussed in detail in Bolatto et al. (2013), departure from the Galactic conversion factor is both observed and expected in starburst galaxies. It has been shown that adopting the Galactic conversion factor would cause the inferred molecular gas mass to exceed the dynamical mass for the central region of a starburst galaxy (Solomon et al. 1997; Downes \& Solomon 1998). Using galaxy merger hydrodynamic simulations, which incorporate dust and molecular line radiative transfer calculations, Narayanan et al.
(2011) have shown that in merger-induced starbursts, the combined effect of increased velocity dispersion and kinetic temperature increases the velocity-integrated $\mathrm{CO}$ intensity, and lowers the $\mathrm{CO}$ conversion factor from the Galactic value by a factor of $\sim 2-10$. The authors also note that in the PSB phase, it is less trivial to simply relate the conversion factor to the gas velocity dispersion and temperature owing to varying physical conditions in the PSB galaxy. Some of their merger simulations returned to a Galactic conversion factor value quickly after the peak of the starburst, while others remained low. Furthermore, if AGN heat the bulk of molecular gas, the conversion factor could be lower from the Galactic value in PSBs with AGN. Note that a lower CO conversion factor than the assumed Galactic value will lower the gas fractions observed in PSBs compared to normal galaxies.

\section{SUMMARY AND CONCLUSIONS}

Using the SMT, we undertook new CO (2-1) observations of 22 green-valley Seyfert PSB candidate galaxies at redshift $z=0.02-$ 0.06 . The sample was selected using the dust-corrected NUV $-g$ colour, $\mathrm{H} \delta$ absorption and $4000 \AA$ break to indicate a PSB signature, and the emission line ratios to indicate Seyfert activity. We analysed our sample with previous samples of 94 PSBs. The combined sample probes a variety of stages in a possible evolutionary sequence, and spans a range of AGN properties. Our main results are as follows.

(i) We detect molecular gas in only 6 out of 22 Seyfert PSB galaxies we targeted. Using comparable sensitivity limits, this is consistent with the detection in PSB sample by French et al. (2015) using the SMT. However, taking into account our upper limits, the mean and the dispersion of the distribution of the gas fraction in our green-valley Seyfert PSBs $(\mu=0.025, \sigma=0.018)$ are much smaller than previous samples of Seyfert PSBs or PSBs in general ( $\mu \sim 0.1-0.2, \sigma \sim 0.1-0.2$; French et al. 2015; Rowlands et al. 2015; Alatalo et al. 2016). This difference may partly be explained by the evolution of molecular gas fraction with PSB age if our sample probes the late stage of the evolution. However, PSBs in French et al. (2015) also have similar green-valley colours but they show a wider range of gas fraction, despite their low SFR inferred from their $\mathrm{H} \alpha$ emission. The comparison between these samples is complicated by the differing selections on $\mathrm{H} \delta$ absorption, as weaker $\mathrm{H} \delta$ is consistent with either a weaker starburst or a later PSB age.

(ii) The distribution of gas fraction in PSBs is significantly different from young star-forming galaxies from COLD GASS survey (Saintonge et al. 2011). PSBs are more likely to have lower gas fraction. The Seyfert PSBs have a distribution of gas fraction that is even more significantly different from that of normal star-forming galaxies of similar $D_{n}(4000)$.

(iii) The WISE flux ratio, $\log f_{12} / f_{4.6}$, is an excellent proxy for gas fraction for both PSBs and non-PSBs. We find a statistically significant $(5 \sigma)$ relationship between $\log f_{12} / f_{4.6}$ and $\log f_{\text {gas }}$ for both PSBs and normal star-forming galaxies.

\section{ACKNOWLEDGEMENTS}

We thank Xavier Prochaska and Ann Zabludoff for their useful comments and suggestions. HY would like to acknowledge support by NSF grants AST-0808133 and AST-1615730. KDF acknowledges support from NSF grant DGE-1143953, PEO, and the ARCS Phoenix Chapter and Burton Family. 


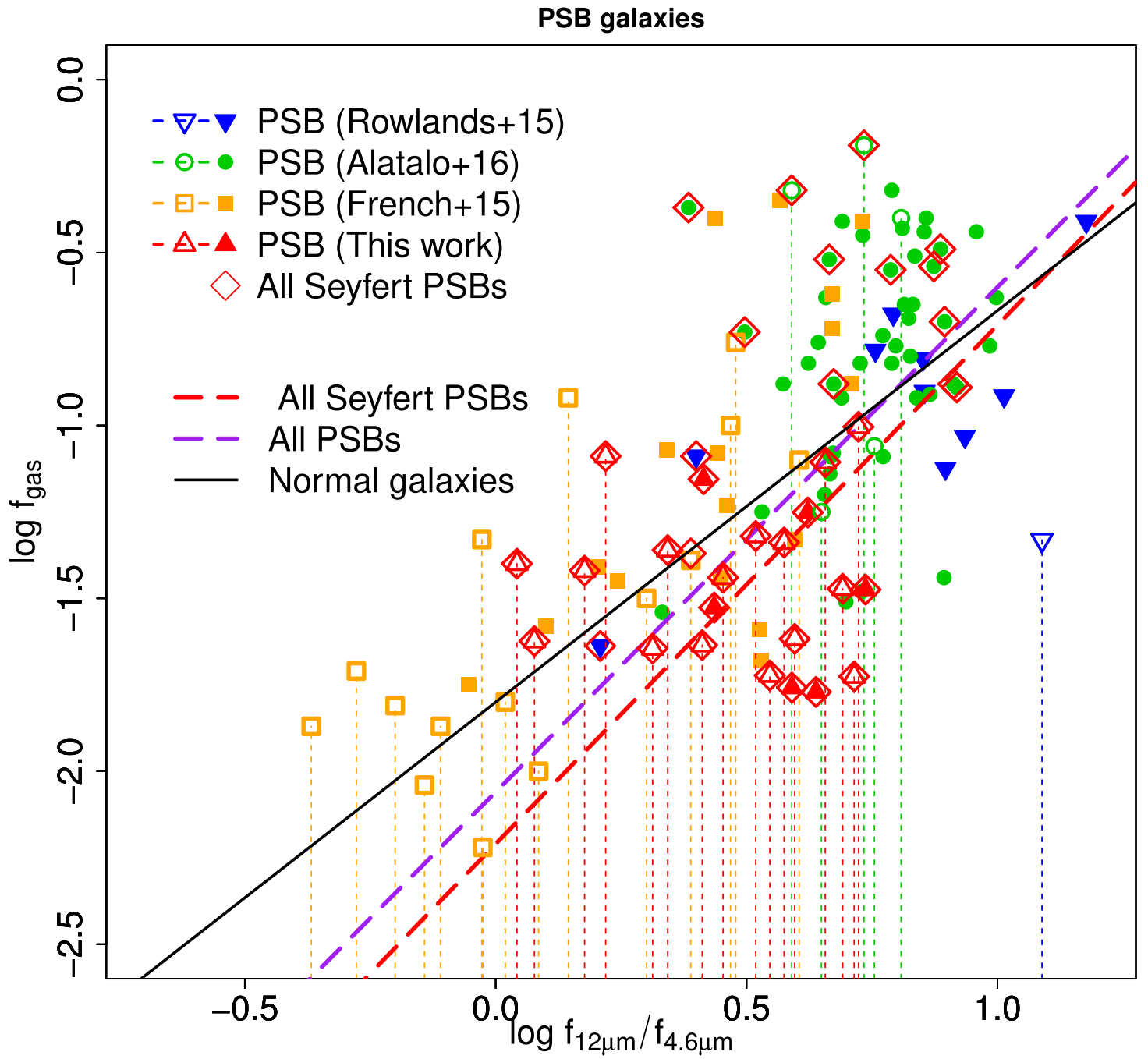

Figure 8. WISE flux ratio $\log f_{12} / f_{4.6}$ versus molecular gas fraction for PSBs (in this work, French et al. 2015; Rowlands et al. 2015; Alatalo et al. 2016). The filled symbols denote PSBs with CO detections while the open symbols (except the purple diamonds) and the dashed lines denote non-detected PSBs and their upper limits. The purple line is the linear fit to the Seyfert PSB data including the upper limits while the cyan line is the fit to all PSB data. The black line is the same as the best-fitting line for non-PSBs in Fig. 7. The slope of the best-fitting line (the correlation) for PSBs is statistically significant (5 $\sigma$ ).

Table 2. Summary statistics of the distribution of gas fraction in PSBs for the new and old CO data. The summary statistics in the table are estimated using the Kaplan-Meier method and therefore take into account the CO non-detections. The percentiles show the probability that the gas fraction of a sample is below the given value. Our late-stage Seyfert sample has a distribution of gas fraction with the lowest mean and standard deviation compared to other samples (French et al. 2015; Rowlands et al. 2015; Alatalo et al. 2016). The French et al. (2015) sample, which may also be late-stage PSBs, has a wider range of gas fraction distribution.

\begin{tabular}{lccccccc}
\hline Study & Mean & Std deviation & 5 per cent & 25 per cent & 50 per cent & 75 per cent & 95 per cent \\
\hline This work (Seyfert) & 0.025 & 0.018 & - & 0.017 & 0.017 & 0.030 & 0.070 \\
French et al. (2015) & 0.081 & 0.122 & - & - & 0.026 & 0.083 & 0.398 \\
Alatalo et al. (2016) & 0.216 & 0.214 & 0.031 & 0.083 & 0.170 & 0.288 & 0.426 \\
Rowlands et al. (2015) & 0.133 & 0.103 & 0.023 & 0.075 & 0.122 & 0.165 & - \\
Seyferts in all works & 0.088 & 0.116 & 0.017 & 0.017 & 0.023 & 0.132 & 0.324 \\
PSBs in all works & 0.133 & 0.176 & - & 0.021 & 0.075 & 0.182 & 0.398 \\
\hline
\end{tabular}

The SMT is operated by the Arizona Radio Observatory (ARO), Steward Observatory and University of Arizona. We thank the operators and staff of the ARO.

Funding for the SDSS and SDSS-II has been provided by the Alfred P. Sloan Foundation, the Participating Institutions, the National Science Foundation, the US Department of Energy, the National
Aeronautics and Space Administration, the Japanese Monbukagakusho, the Max Planck Society and the Higher Education Funding Council for England. The SDSS website is http://www.sdss.org/.

The SDSS is managed by the Astrophysical Research Consortium for the Participating Institutions. The Participating Institutions are the American Museum of Natural History, Astrophysical 
Institute Potsdam, University of Basel, University of Cambridge, Case Western Reserve University, University of Chicago, Drexel University, Fermilab, the Institute for Advanced Study, the Japan Participation Group, Johns Hopkins University, the Joint Institute for Nuclear Astrophysics, the Kavli Institute for Particle Astrophysics and Cosmology, the Korean Scientist Group, the Chinese Academy of Sciences (LAMOST), Los Alamos National Laboratory, the Max-Planck-Institute for Astronomy (MPIA), the MaxPlanck-Institute for Astrophysics (MPA), New Mexico State University, Ohio State University, University of Pittsburgh, University of Portsmouth, Princeton University, the United States Naval Observatory and the University of Washington.

\section{REFERENCES}

Aihara H. et al., 2011, ApJS, 193, 29

Akritas M. G., Murphy S. A., LaValley M. P., 1995, J. Am. Stat. Assoc., 90, 170

Alatalo K. et al., 2011, ApJ, 735, 88

Alatalo K., Cales S. L., Appleton P. N., Kewley L. J., Lacy M., Lisenfeld U., Nyland K., Rich J. A., 2014, ApJ, 794, L13

Alatalo K. et al., 2016, ApJ, 827, 106

Assef R. J. et al., 2013, ApJ, 772, 26

Baldwin J. A., Phillips M. M., Terlevich R., 1981, PASP, 93, 5

Balogh M. L., Morris S. L., Yee H. K. C., Carlberg R. G., Ellingson E., 1999, ApJ, 527, 54

Barnes J. E., Hernquist L. E., 1991, ApJ, 370, L65

Barro G. et al., 2013, ApJ, 765, 104

Bolatto A. D., Wolfire M., Leroy A. K., 2013, ARA\&A, 51, 207

Boselli A., Cortese L., Boquien M., Boissier S., Catinella B., Lagos C., Saintonge A., 2014, A\&A, 564, A66

Bruzual A. G., 1983, ApJ, 273, 105

Bruzual G., Charlot S., 2003, MNRAS, 344, 1000

Cen R., 2012, ApJ, 755, 28

Charlot S., Fall S. M., 2000, ApJ, 539, 718

Cheung E. et al., 2012, ApJ, 760, 131

Cicone C. et al., 2014, A\&A, 562, A21

Couch W. J., Sharples R. M., 1987, MNRAS, 229, 423

Cox D. R., 1972, J. R. Stat. Soc., 34, 187

Davis T. A. et al., 2014, MNRAS, 444, 3427

De Lucia G., Poggianti B. M., Halliday C., Milvang-Jensen B., Noll S., Smail I., Zaritsky D., 2009, MNRAS, 400, 68

Dekel A., Burkert A., 2014, MNRAS, 438, 1870

Di Matteo T., Springel V., Hernquist L., 2005, Nature, 433, 604

Donley J. L. et al., 2012, ApJ, 748, 142

Donoso E. et al., 2012, ApJ, 748, 80

Downes D., Solomon P. M., 1998, ApJ, 507, 615

Dressler A., Gunn J. E., 1983, ApJ, 270, 7

Dressler A., Oemler A., Jr, Poggianti B. M. Gladders M. D., Abramson L., Vulcani B., 2013, ApJ, 770, 62

Fabello S., Kauffmann G., Catinella B., Giovanelli R., Haynes M. P., Heckman T. M., Schiminovich D., 2011, MNRAS, 416, 1739

Faber S. M. et al., 2007, ApJ, 665, 265

Fang J. J., Faber S. M., Salim S., Graves G. J., Rich R. M., 2012, ApJ, 761, 23

Feigelson E. D., Nelson P. I., 1985, ApJ, 293, 192

Feruglio C., Maiolino R., Piconcelli E., Menci N., Aussel H., Lamastra A., Fiore F., 2010, A\&A, 518, L155

Fischer J. et al., 2010, A\&A, 518, L41

French K. D., Yang Y., Zabludoff A., Narayanan D., Shirley Y., Walter F., Smith J.-D., Tremonti C. A., 2015, ApJ, 801, 1

García-Burillo S. et al., 2014, A\&A, 567, A125

Geréb K., Morganti R., Oosterloo T. A., Hoppmann L., Staveley-Smith L., 2015, A\&A, 580, A43

Gladders M. D., López-Cruz O., Yee H. K. C., Kodama T., 1998, ApJ, 501, 571
Halsel D., 2012, Statistics for Censored Environmental Data, 2 edn. Wiley, New York

Harrington D. P., Fleming T. R., 1982, Biometrika, 69, 553

Hayward C. C. et al., 2014, MNRAS, 445, 1598

Hopkins P. F., Hernquist L., 2009, ApJ, 698, 1550

Hopkins P. F., Hernquist L., Cox T. J., Di Matteo T., Robertson B., Springel V., 2006, ApJS, 163, 1

Hopkins P. F., Hernquist L., Cox T. J., Kereš D., 2008, ApJS, 175, 356

Huang M.-L., Kauffmann G., 2014, MNRAS, 443, 1329

Kaplan E. L., Meier P. M., 1958, J. Am. Stat. Assoc., 53, 457

Kauffmann G. et al., 2003, MNRAS, 346, 1055

Kaviraj S., Kirkby L. A., Silk J., Sarzi M., 2007, MNRAS, 382, 960

Kewley L. J., Dopita M. A., Sutherland R. S., Heisler C. A., Trevena J., 2001, ApJ, 556, 121

Klein J. P., Moeschberger M. L., 2005, Survival Analysis: Techniques for Censored and Truncated Data. Springer-Verlag, New York

Kormendy J., 2013, in Thomas D., Pasquali A., Ferreras I., eds, Proc. IAU Symp. 295, The Intriguing Life of Massive Galaxies. Cambridge Univ. Press, Cambridge, p. 241

Lamastra A., Bianchi S., Matt G., Perola G. C., Barcons X., Carrera F. J., 2009, A\&A, 504, 73

Lee L., Lee M. L., 2015, Package NADA. Available at: https://cran. r-project.org/web/packages/NADA/index.html

Leroy A. K. et al., 2013, AJ, 146, 19

Mantel N., 1966, Cancer Chemother. Rep, 50, 163

Martin D. C. et al., 2005, ApJ, 619, L1

Martinez R. L. M. C., 2007, Diagnostics for Choosing between Log-rank and Wilcoxon Tests, Dissertations, 895. Available at: http://scholarworks. wmich.edu/dissertations/895

Murray N., Quataert E., Thompson T. A., 2005, ApJ, 618, 569

Narayanan D. et al., 2008, ApJS, 176, 331

Narayanan D., Krumholz M., Ostriker E. C., Hernquist L., 2011, MNRAS, 418,664

Pandya V. et al., 2016, MNRAS, preprint (arXiv:1611.03869)

Pawlik M. M., Wild V., Walcher C. J., Johansson P. H., Villforth C., Rowlands K., Mendez-Abreu J., Hewlett T., 2016, MNRAS, 456, 3032

Poggianti B. M., Smail I., Dressler A., Couch W. J., Barger A. J., Butcher H., Ellis R. S., Oemler A., Jr, 1999, ApJ, 518, 576

Rowlands K., Wild V., Nesvadba N., Sibthorpe B., Mortier A., Lehnert M., da Cunha E., 2015, MNRAS, 448, 258

Rupke D. S., Veilleux S., Sanders D. B., 2002, ApJ, 570, 588

Saintonge A. et al., 2011, MNRAS, 415, 32

Saintonge A. et al., 2012, ApJ, 758, 73

Sanders D. B., Soifer B. T., Elias J. H., Madore B. F., Matthews K., Neugebauer G., Scoville N. Z., 1988, ApJ, 325, 74

Sandstrom K. M. et al., 2013, ApJ, 777, 5

Scannapieco E., Oh S. P., 2004, ApJ, 608, 62

Schawinski K., Thomas D., Sarzi M., Maraston C., Kaviraj S., Joo S.-J., Yi S. K., Silk J., 2007, MNRAS, 382, 1415

Schawinski K. et al., 2009, ApJ, 690, 1672

Schawinski K. et al., 2014, MNRAS, 440, 889

Sen P. K., 1968, J. Am. Stat. Assoc., 63, 1379

Silk J., Nusser A., 2010, ApJ, 725, 556

Silk J., Rees M. J., 1998, A\&A, 331, L1

Snyder G. F., Cox T. J., Hayward C. C., Hernquist L., Jonsson P., 2011, ApJ, 741, 77

Solomon P. M., Downes D., Radford S. J. E., Barrett J. W., 1997, ApJ, 478, 144

Spoon H. W. W. et al., 2013, ApJ, 775, 127

Springel V., Di Matteo T., Hernquist L., 2005, MNRAS, 361, 776

Sturm E. et al., 2011, ApJ, 733, L16

Sun A.-L., Greene J. E., Zakamska N. L., Nesvadba N. P. H., 2014, ApJ, 790,160

Toomre A., Toomre J., 1972, ApJ, 178, 623

Utomo D., Kriek M., Labbé I., Conroy C., Fumagalli M., 2014, ApJ, 783, L30

Veilleux S. et al., 2013, ApJ, 776, 27 
Wild V., Walcher C. J., Johansson P. H., Tresse L., Charlot S., Pollo A., Le Fèvre O., de Ravel L., 2009, MNRAS, 395, 144

Wild V., Heckman T., Charlot S., 2010, MNRAS, 405, 933

Wild V., Charlot S., Brinchmann J., Heckman T., Vince O., Pacifici C., Chevallard J., 2011, MNRAS, 417, 1760

Wild V., Almaini O., Dunlop J., Simpson C., Rowlands K., Bowler R., Maltby D., McLure R., 2016, MNRAS, 463, 832

Wong O. I. et al., 2012, MNRAS, 420, 1684

Woo J., Dekel A., Faber S. M., Koo D. C., 2015, MNRAS, 448, 237

Wright E. L. et al., 2010, AJ, 140, 1868

Yesuf H. M., Faber S. M., Trump J. R., Koo D. C., Fang J. J., Liu F. S., Wild V., Hayward C. C., 2014, ApJ, 792, 84

Young L. M. et al., 2011, MNRAS, 414, 940

Zabludoff A. I., Zaritsky D., Lin H., Tucker D., Hashimoto Y., Shectman S. A., Oemler A., Kirshner R. P., 1996, ApJ, 466, 104

Zubovas K., King A., 2012, ApJ, 745, L34

\section{APPENDIX}

This appendix presents materials that support the analysis in the main sections of the paper.

\section{A1 Evolution of $\mathrm{H} \delta$ equivalent width after a starburst}

Fig. A1 shows the evolution of $\mathrm{H} \delta$ equivalent width for Bruzual \& Charlot (2003) starburst models, with a star formation time-
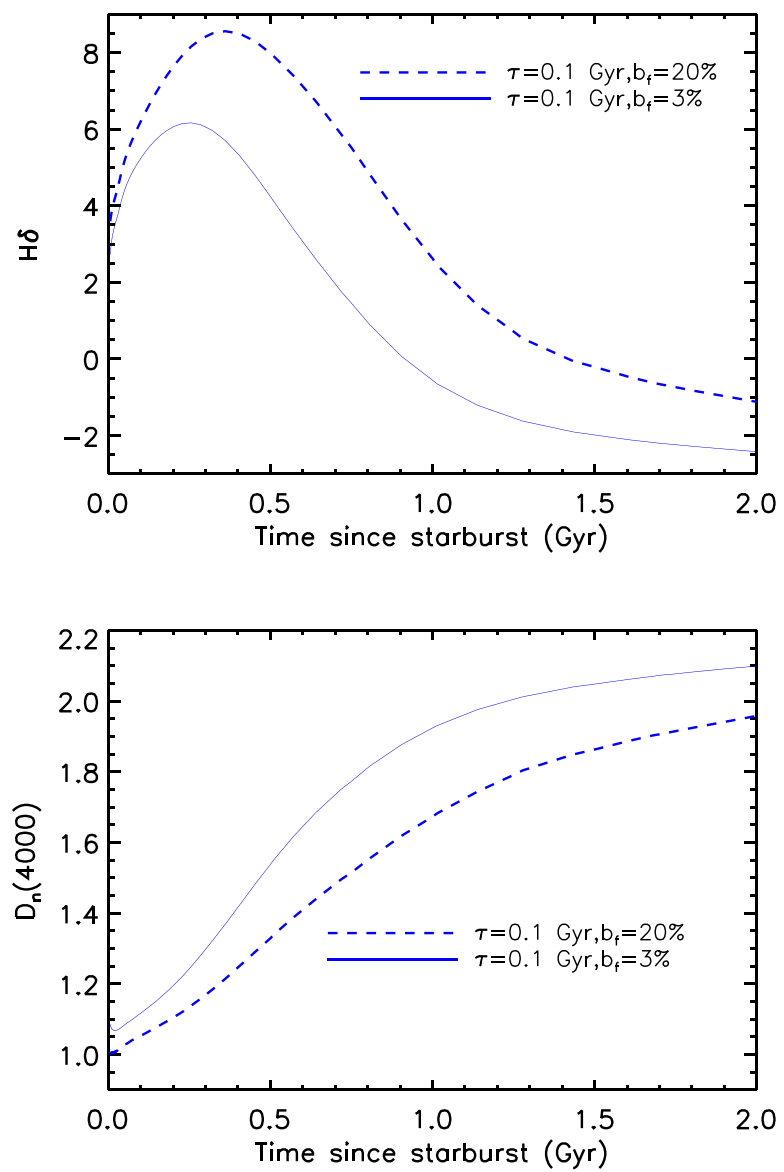

Figure A1. The evolution the $\mathrm{H} \delta$ absorption and $D_{n}(4000)$ with time since the starburst for the Bruzual \& Charlot (2003) burst model tracks with a star formation time-scale, $\tau=0.1 \mathrm{Gyr}$, and a burst mass fraction $\left(b_{\mathrm{f}}\right) 3$ or 20 per cent.
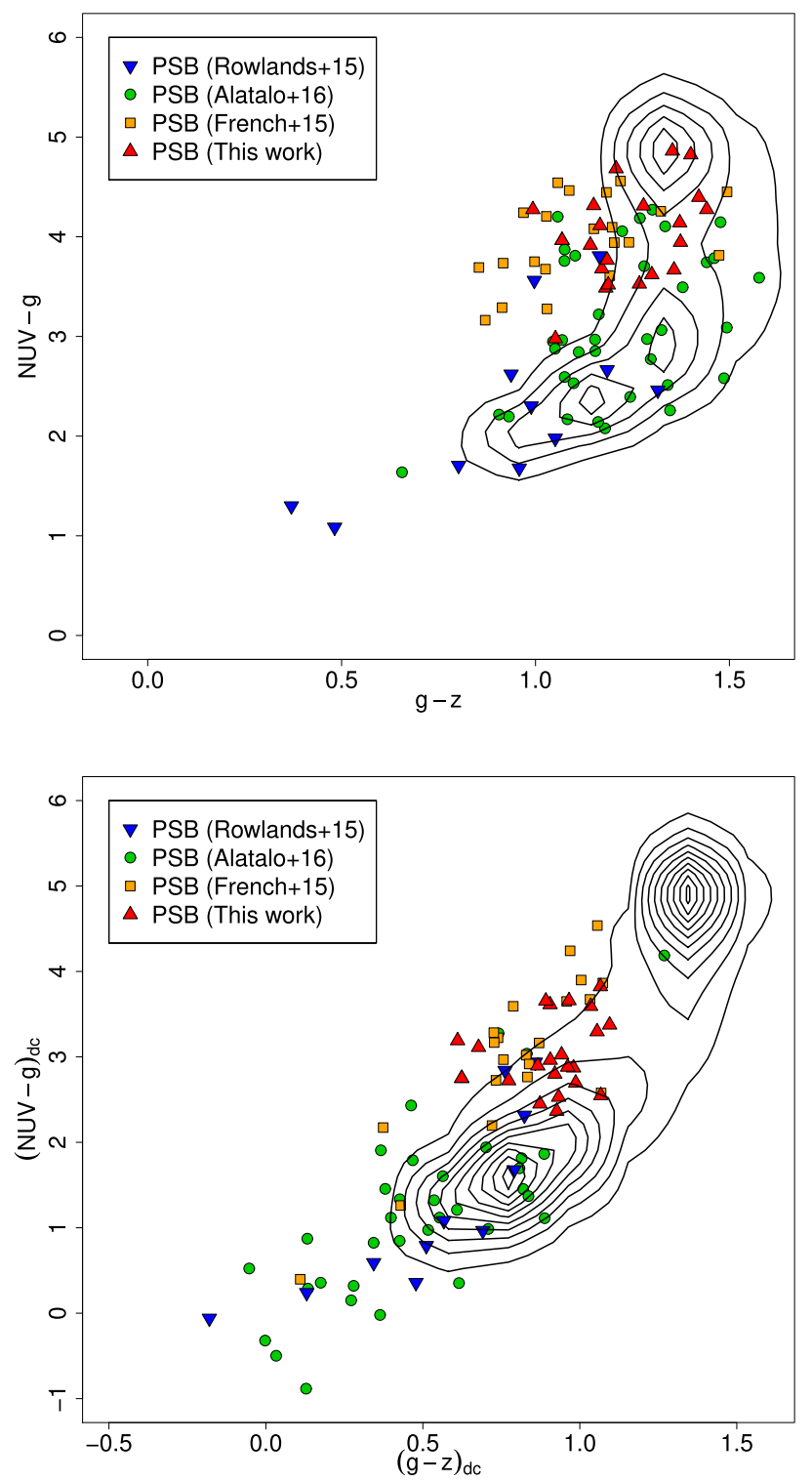

Figure A2. The top panel shows the two-colour plot of NUV $-g$ and $g-z$ before the dust correction and the bottom panel shows the two-colour plot after the dust correction. The coloured points are PSBs (in this work, French et al. 2015; Rowlands et al. 2015; Alatalo et al. 2016). The contours represent the number density of SDSS galaxies at redshift $z=0.02-0.06$ with stellar mass $M=10^{10}-10^{11} \mathrm{M}_{\odot}$. The positions of our Seyfert PSBs in the diagrams indicate that they are not dusty star-forming galaxies rather they are transitional galaxies in green valley.

scale, $\tau=0.1$ Gyr and a burst mass fraction $\left(b_{\mathrm{f}}\right) 3$ or 20 per cent. As detailed in Yesuf et al. (2014), the $\mathrm{H} \delta$ is dependent on the magnitude of the burst and there is generally a degeneracy between the burst mass fraction and the burst time-scale. The figure demonstrates, with particular models above, that late-stage PSBs can have $\mathrm{H} \delta<3 \AA$, which is lower than the threshold often used to define PSBs.

\section{A2 A comparison of our PSB sample with previous samples}

This section presents additional figures that compare the stellar population of our Seyfert PSBs with previous samples of PSBs (French et al. 2015; Rowlands et al. 2015; Alatalo et al. 2016). Fig. A2 


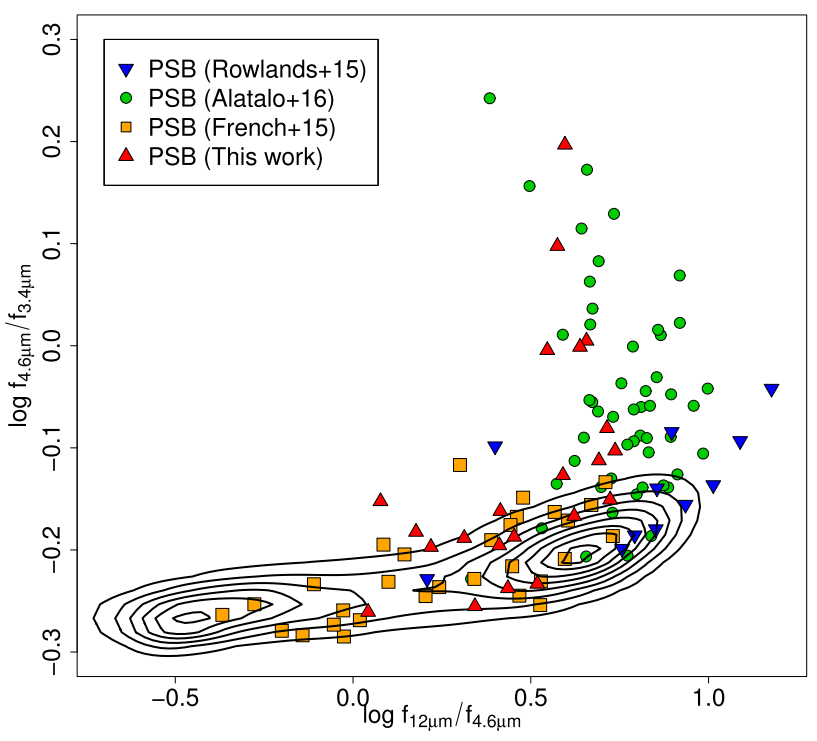

Figure A3. The WISE two-colour diagram. $\log f_{12} / f_{4.6}$ is indicator of sSFR and while the $\log f_{4.6} / f_{3.4}$ is indicator of hot dust emission by AGN or starburst or both. The contours denote the distribution of bimodel galaxy population at $z=0.02-0.06$ and $\log M\left(\mathrm{M}_{\odot}\right)=10-11$. The blue cloud is the density cloud with peak at $\log f_{12} / f_{4.6} \sim 0.6$ while the red sequence is the density cloud with peak at $\log f_{12} / f_{4.6} \sim-0.45$. PSBs show a wide range in $\log f_{12} / f_{4.6}$, and those with $\log f_{4.6} / f_{3.4}>-0.06$ may be AGN.

shows the NUV $-g$ versus $g-z$ colours before and after the dust correction. Before the dust correction, star-forming galaxies form a diagonal track that stretches from blue to red colours. The red end of this track is inhabited by dusty galaxies. The quiescent galaxies form a separate concentration above the dusty star-forming galaxies. The locations our Seyfert PSBs in the two-colour diagrams indicate that they are not dusty star-forming galaxies but are transitional galaxies in green valley. The dust-corrected diagram in addition shows that stellar ages of our PSBs and the PSBs in French et al. (2015) are comparable and the PSBs in Rowlands et al. (2015) and Alatalo et al. (2016) are younger than our sample. Fig. A3 depicts $\log f_{12} / f_{4.6}$ versus $\log f_{4.6} / f_{3.4}$ (Wright et al. 2010). Stellar populations younger than $0.6 \mathrm{Gyr}$ dominate the $12 \mu \mathrm{m}$ emission and $\log f_{12} / f_{4.6}$ is known to correlate well with sSFR (Donoso et al. 2012) and $\log f_{4.6} / f_{3.4}$ is sensitive to hot dust emission from AGN. Normal galaxies form a tight bimodal sequence with some vertical scatter. Star-forming galaxies are located at high $\log f_{12} / f_{4.6}$, while quiescent galaxies are located at $\operatorname{low} \log f_{12} / f_{4.6}$.

Fig. A4 depicts the WISE flux ratio $\log f_{4.6} / f_{3.4}$ versus $\log f_{\text {gas }}$ for PSBs. The $\log f_{4.6} / f_{3.4}>-0.06$ criterion can identify hot dust emission from AGN albeit only with $\sim 50$ per cent reliability (Assef

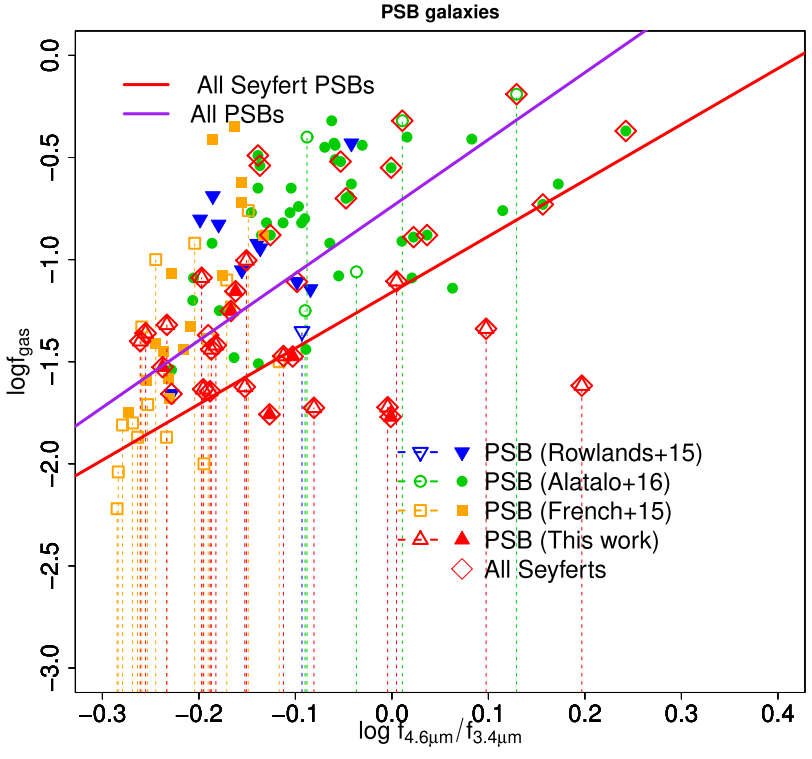

Figure A4. WISE flux ratio $\log f_{4.6} / f_{3.4}$ versus molecular gas fraction for PSBs (in this work, French et al. 2015; Rowlands et al. 2015; Alatalo et al. 2016). The observed statistically significant trend of decreasing gas fraction with $\log f_{4.6} / f_{3.4}$ is consistent with stellar dust heating process that decreases with the starburst age, and it is inconsistent with AGN feedback that happens close to the starburst phase.

et al. 2013). Anti-correlation between $\log f_{4.6} / f_{3.4}$ and $\log f_{\text {gas }}$ may naively be expected if AGN feedback destroys the molecular gas by heating it. Such a trend is not seen in the data. There is instead somewhat significant $(\sim 2 \sigma)$ correlation between $\log f_{4.6} / f_{3.4}$ and $\log f_{\text {gas }}$ for the BPT-identified Seyfert PSBs. The current data cannot rule out AGN feedback, and the observed trend is consistent with delayed AGN feedback, which is inefficient at earlier times when the molecular gas fraction is above $\sim 10$ per cent. Future study with large number of PSBs with $\log f_{4.6} / f_{3.4}>-0.06$ may constrain this model better. It should be noted that correlation between $\log f_{4.6} / f_{3.4}$ and $\log f_{\text {gas }}$ for all PSBs in the combined sample is highly significant $(>5 \sigma)$ and it is likely tracing stellar dust heating process that declines with the starburst age.

\section{A3 CO luminosities of Seyfert PSBs}

Table A1 presents the observed CO luminosities of our new Seyfert PSB sample or $3 \sigma$ upper limits in the case of no detections. Note that TPSB2 and TPSB23 are the same object, which is observed twice and we have combined the two observations in the analysis presented in the main text of the paper. 
Table A1. CO (2-1) line luminosities and full width at half-maximum (FWHM) from fitting a Gaussian to the lines.

\begin{tabular}{|c|c|c|c|c|}
\hline Target & $\begin{array}{c}\text { RA } \\
\text { (degree) }\end{array}$ & $\begin{array}{c}\text { Dec. } \\
\text { (degree) }\end{array}$ & $\begin{array}{c}L_{\mathrm{CO}}^{\prime} \\
\left(10^{7} \mathrm{~K} \mathrm{~km} \mathrm{~s}^{-1} \mathrm{pc}^{2}\right)\end{array}$ & $\begin{array}{c}\text { FWHM } \\
\left(\mathrm{km} \mathrm{s}^{-1}\right)\end{array}$ \\
\hline TPSB1 & 212.01667 & 7.3276444 & $14.17 \pm 2.74$ & $109.8 \pm 13.8$ \\
\hline TPSB2 & 134.61917 & 0.02346944 & $21.5222 \pm 4.95$ & $234.8 \pm 54.0$ \\
\hline TPSB4 & 182.01942 & 55.407672 & $<35.36$ & - \\
\hline TPSB5 & 173.41283 & 52.674611 & $<29.27$ & - \\
\hline TPSB6 & 170.94588 & 35.442308 & $<21.61$ & - \\
\hline TPSB7 & 203.56175 & 34.194147 & $9.59 \pm 2.55$ & $201.7 \pm 48.3$ \\
\hline TPSB8 & 189.51733 & 48.345097 & $<14.97$ & - \\
\hline TPSB9 & 117.96617 & 49.814314 & $44.81 \pm 11.92$ & $137.8 \pm 22.2$ \\
\hline TPSB10 & 180.51921 & 35.321681 & $<17.77$ & - \\
\hline TPSB11 & 137.87483 & 45.468278 & $19.60 \pm 4.72$ & $370.2 \pm 169.2$ \\
\hline TPSB12 & 139.49937 & 50.002175 & $<20.09$ & - \\
\hline TPSB13 & 173.16771 & 52.950400 & $<11.08$ & - \\
\hline TPSB14 & 178.62254 & 42.980203 & $<11.86$ & - \\
\hline TPSB15 & 179.02850 & 59.424919 & $<16.27$ & - \\
\hline TPSB16 & 190.45025 & 47.708878 & $<14.73$ & - \\
\hline TPSB17 & 198.74925 & 51.272583 & $<10.41$ & - \\
\hline TPSB18 & 200.95183 & 43.301181 & $37.94 \pm 5.70$ & $436.3 \pm 125.8$ \\
\hline TPSB19 & 236.93392 & 41.402294 & $<23.06$ & - \\
\hline TPSB20 & 240.65804 & 41.293433 & $<21.51$ & - \\
\hline TPSB21 & 247.63600 & 39.384192 & $<15.28$ & - \\
\hline TPSB23 & 134.61913 & 0.02346944 & $15.20 \pm 4.18$ & $126.1 \pm 24.0$ \\
\hline TPSB24 & 145.18546 & 21.234358 & $<9.23$ & - \\
\hline TPSB26 & 172.08300 & 27.622097 & $<17.80$ & - \\
\hline TPSB28 & 222.65771 & 22.734336 & $<6.82$ & - \\
\hline
\end{tabular}

This paper has been typeset from a $\mathrm{T}_{\mathrm{E}} \mathrm{X} / \mathrm{L} \mathrm{T} \mathrm{E} \mathrm{X}$ file prepared by the author. 